\title{
ЭКОНОМИКО-МАТЕМАТИЧЕСКОЕ МОДЕЛИРОВАНИЕ
}

УДК $330.4+338.2$

\author{
С. М. Иващенко
}

\section{МНОГОСЕКТОРНАЯ МОДЕЛЬ ДИНАМИЧЕСКОГО СТОХАСТИЧЕСКОГО ОБЩЕГО ЭКОНОМИЧЕСКОГО РАВНОВЕСИЯ РОССИЙСКОЙ ЭКОНОМИКИ}

\begin{abstract}
В статье разработана многосекторная модель динамического стохастического общего экономического равновесия (ДСОЭР), делящая производство на 5 секторов: 1)добыча полезных ископаемых; 2) обрабатывающие производства; 3) производство и распределение электроэнергии, газа и воды; 4) торговля, транспорт и связь; 5) другое. Параметры модели оценены по 29 рядам российской статистики. Модель демонстрирует высокое качество прогнозов вне выборки, превосходящее показатели авторегрессионных моделей. Рассчитаны последствия снижения экспортных цен, ограничения доступности внешних займов, ужесточения денежной политики, а также роста государственных расходов. Библиогр. 18 назв. Ил. 5. Табл. 1.

Ключевые слова: ДСОЭР, DSGE, отрасли, прогнозы вне выборки.
\end{abstract}

Sergey M. Ivashchenko

\section{MULTIPLE SECTORS DSGE MODEL OF RUSSIA}

A dynamic stochastic general equilibrium model with multiple sectors is constructed. The production is divided into 5 sectors: 1 ) mining; 2) manufacturing; 3 ) electricity, gas and water; 4) trade, transport and communication; 5) other. The model is estimated in 29 time-series of Russian statistical data. The model produces high quality out-of-sample forecasts (better than auto regressions). The consequences of export price decline, restriction of access to foreign finance, tighter monetary policy and higher government spending are computed. Refs 18. Figs 5. Table 1.

Keywords: DSGE; industries; out of sample forecasts.

\section{Введение}

Динамические стохастические модели общего экономического равновесия (ДСОЭР) являются одним из основных инструментов макроэкономического анализа, которые широко применяются центральными банками и другими офици-

Сергей Михайлович ИВАЩЕНКО - Санкт-Петербургский экономико-математический институт РАН, ул. Серпуховская 36-38, Санкт-Петербург, 190013, Российская Федерация; glucke_ru@ pisem.net; sergey.ivashchenko.ru@gmail.com

Sergey M.IVASHCHENKO - St. Petersburg Economics and Mathematics Institute of the Russian Academy of Sciences, 36-38 Serpukhovskaya str., St. Petersburg, 190013, Russian Federation; glucke_ru@ pisem.net; sergey.ivashchenko.ru@gmail.com

( С Санкт-Петербургский государственный университет, 2016 
альными организациями многих стран [Tovar, 2009]. Данный класс моделей продемонстрировал высокое качество прогнозов, что позволяет сопоставить их с авторегрессионными моделями [Adolfson, Linde, Villani, 2007; Rubaszek, Skrzypczynski 2008; del Negro and Schorfheide, 2012; Иващенко, 2013], а во многих случаях даже говорить об их превосходстве. Основное достоинство ДСОЭР-моделей заключается в том, что они объясняют динамику переменных в терминах теоретической модели (основанной на предпочтениях и технологиях) и позволяют обойти проблему критики Лукаса [Lucas, 1976].

Модели межотраслевого баланса (МОБ) являются другим классом моделей, который отражает неоднородность экономики (деление на отрасли) и взаимосвязь отраслей. Модели, относящиеся к этому классу, возникнув в середине XX века, продолжают развиваться. Указанные модели позволили сформировать систему сбора статистики (таблицы «затраты - выпуск»). В результате появились работы, использующие таблицы «затраты - выпуск» для получения оценок параметров, присутствующих в других моделях [Lombardo, Ravenna, 2012]. Однако многие работы направлены на расширение МОБ-моделей и формирование соответствующей статистики [O’Doherty, Tol, 2007]. Недостатки этого подхода состоят в том, что конечный спрос имеет экзогенный характер, а также наблюдается непостоянство коэффициентов модели.

ДСОЭР-модели не проводят разделения экономики на отрасли производства. Чаще всего встречаются модели с одним или двумя секторами (торгуемых и не торгуемых товаров) производства [Rubaszek, Skrzypczynski, 2008; Rudolf, Zurlinden, 2014]. Реже встречаются модели с добавлением энергетического сектора [Ojeda, Parra-Polania, Vargas, 2014].

Всего несколько моделей включают в себя множество секторов [Carvalho, Lee, 2011; Lee, 2010]. Однако в модели [Lee, 2010] существует различие секторов в степени негибкости ценообразования и долях спроса, приходящихся на сектор, отсутствует взаимодействие секторов. Модель [Carvalho, Lee, 2011] имеет больший уровень неоднородности секторов: различные коэффициенты общей производительности факторов и использование товаров других секторов в производственной функции. Тем не менее единая форма кривой спроса для всех секторов не позволяет воспроизвести эффект, наблюдающийся в моделях МОБ, когда поддержка одного сектора ведет к росту в некоторых других и не влияет на третьи. Кроме того, в модели отсутствует капитал.

Количество ДСОЭР-моделей российской экономики относительно невелико. Есть модели, включающие цены на нефть, но лишь в качестве источника экзогенного дохода домохозяйств, приходящего со стороны внешнего сектора [Malakhovskaya, Minabutdinov, 2014]. В модели [Дробышевский, Полбин, 2015] экзогенное производство нефти используется как один из факторов производства, а часть дохода идет в государственный бюджет. Однако в этих моделях производство нефти не связано с использованием каких-либо факторов производства и нет отраслевой структуры экономики. К тому же приведенные выше работы не содержат показателей, характеризующих качество описания статистических данных указанными моделями. Модель [Иващенко, 2013] имеет такое описание, но в ней отсутствует разбиение экономики на отрасли (несмотря на наличие банковского сектора). 
Целью настоящей статьи является анализ последствий ряда событий последних лет (представляющих собой долгосрочное изменение поведения отдельных переменных), которые можно интерпретировать как изменение политики (в отличие от обычных краткосрочных колебаний). Для достижения цели исследования были решены три задачи. Первой задачей работы является построение ДСОЭР-модели России, качественно описывающей статистические данные, включая неоднородность динамики секторов (в том числе добывающего сектора). Вторая задача заключается в анализе качества построенной модели на основе качества прогнозов вне выборки. Третья задача - это расчет последствий некоторых изменений политики государства и внешнего сектора и сравнение их с последствиями аналогичных воздействий экзогенных шоков.

В данной статье строится ДСОЭР-модель, обладающая высокой степенью неоднородности секторов. Предполагается наличие экзогенного внешнего сектора и доступ фирм, входящих в любой сектор, к внутреннему и внешнему финансовому рынкам. Параметры модели оценены методом максимального правдоподобия по российским данным. На основе использования полученных оценок параметров произведена оценка последствий изменения политики.

\section{1. Модель}

Разработанная ДСОЭР-модель представляет собой модель открытой экономики с 8 группами агентов: домохозяйства, фирмы-производители для 5 отраслей: 1) добыча полезных ископаемых; 2) обрабатывающие производства; 3) производство и распределение электроэнергии, газа и воды; 4) торговля, транспорт и связь; 5) другие, государство и внешний сектор. Агенты взаимодействуют на рынках труда, долговых инструментов в национальной и иностранной валютах, акций и товаров. Схема модели приведена на рис. 1.

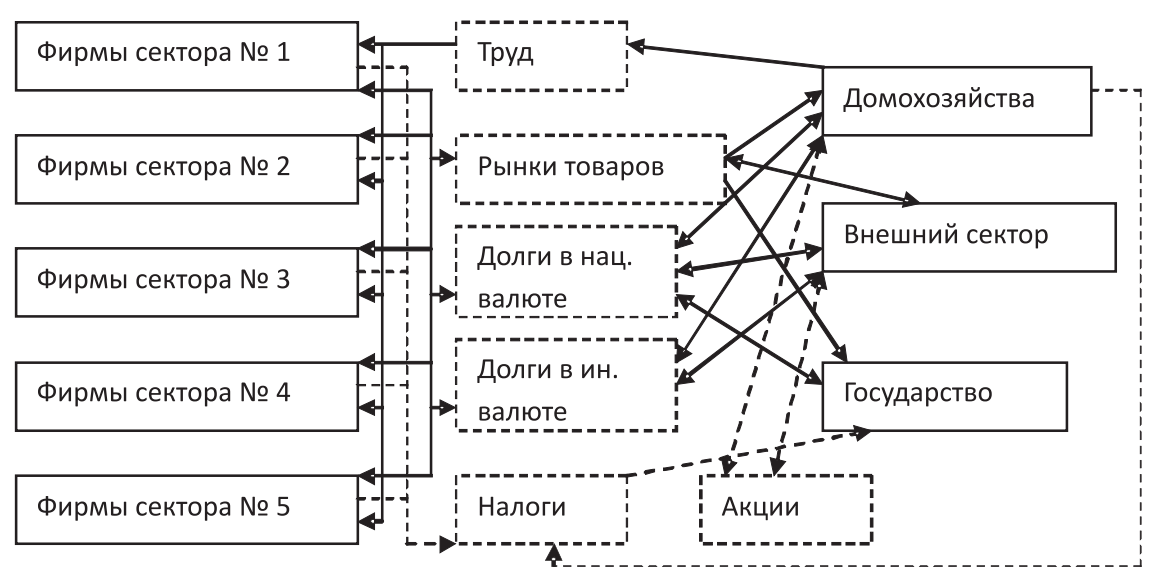

Рис. 1. Структура ДСОЭР-модели

Большинство переменных моделей нестационарны, соответственно при расчетах производится переход к стационарным переменным (см. приложение, табл. А1). Обозначения экзогенных процессов начинается с буквы $z$. Именно они 
вносят стохастику в модель. Система налогообложения состоит из налогов на труд и на выпуск аналогично [Иващенко, 2013].

\section{1. Домохозяйства}

Домохозяйства решают следующую оптимизационную задачу с ограничениями:

$$
\begin{gathered}
E\left(\sum_{t=0}^{\infty} Z_{\beta, t}\left(\begin{array}{l}
\left.\left.\frac{\left(C_{t}-h_{C} H_{t-1}\right)^{1-\omega_{C}}}{1-\omega_{C}}-Z_{L, t} \frac{L_{t}^{1+\omega_{L}}}{1+\omega_{L}}-Z_{M, t}\left(L N\left(\frac{M_{t}}{C_{t} P_{C, t}}\right)-\mu_{M}\right)^{2}\right)\right) \rightarrow \max _{B, C, L, M, X} \\
-Z_{B H, t}\left(\frac{B_{H, t}}{P_{t} Z_{t}}-\mu_{B}\right)^{2}-Z_{B W H, t}\left(\frac{F_{t} B_{W H, t}}{P_{t} Z_{t}}-\mu_{W}\right)^{2}
\end{array}\right)\right) \\
\quad P_{C, t} C_{t}+M_{t}+B_{H, t}+F_{t} B_{W H, t}+\sum_{i=1}^{5} X_{i, H, t} S_{i, t}=\left(1-\tau_{L, t}\right) W_{t} L_{t}+M_{t-1}+ \\
+R_{H, t-1} B_{H, t-1}+F_{t} R_{W, t-1} B_{W H, t-1}+\sum_{i=1}^{5} X_{i, H, t-1}\left(S_{i, t}+D_{i, t}\right)+T_{T R, t},
\end{gathered}
$$

где $C_{t}$ - потребление в периоде $t ; L_{t}-$ предложение труда в периоде $t ; M_{t}-$ запас денег в периоде $t ; P_{C, t}$ - цена потребительских товаров в периоде $t ; B_{H, t}-$ стоимость облигаций в национальной валюте, приобретенных домохозяйствами в периоде $t ; B_{W H, t}$ - стоимость облигаций в иностранной валюте, приобретенных домохозяйствами в периоде $t ; S_{i, t}$ - цена акций отрасли $i$ в периоде $t ; X_{i, H, t}-$ количество акций отрасли $i$, приобретенных домохозяйствами в периоде $t ; \tau_{L, t}-$ ставка налога на труд в периоде $t$; $T_{T R, t}-$ трансферт от государства домохозяйствам в периоде $t$; $R_{H, t}-$ ставка по облигациям в национальной валюте в периоде $t ; R_{W, t}-$ ставка по облигациям в иностранной валюте в периоде $t$; $D_{i, t}$ - дивиденды от фирм сектора $i$ в периоде $t$ и $F_{t}$ - обменный курс в периоде $t$.

Домохозяйства максимизируют ожидаемую дисконтированную функцию полезности (1) при условии бюджетного ограничения (2). Домохозяйства не владеют реальным капиталом, но могут вкладывать средства в акции (фирм из каждой отрасли) и облигации (в национальной и иностранной валютах). Функция полезности состоит из склонности к потреблению с эффектом привычного уровня потребления (habit), несклонности к труду, несклонности объема хранимых денег отличаться от заданного уровня, несклонности позиции в облигациях отходить от заданного уровня. Эта функция полезности аналогична использованной в модели [Иващенко, 2013]. Она содержит комбинацию основных способов введения денег в модель (cash in advance и «деньги в функции полезности»). А негибкость позиции в облигациях является механизмом ограничения долгов, работающим в рамках линейной аппроксимации.

\section{2. Фирмы}

Фирмы сектора $i$ решают следующую оптимизационную задачу с ограничениями: 


$$
\begin{aligned}
& E\left(\sum_{t=0}^{\infty} Z_{\beta, t}\left(\begin{array}{l}
\left.\frac{\left(C_{t}-h_{C} H_{t-1}\right)^{1-\omega_{C}}}{1-\omega_{C}}-Z_{L, t} \frac{L_{t}^{1+\omega_{L}}}{1+\omega_{L}}-Z_{M, t}\left(L N\left(\frac{M_{t}}{C_{t} P_{C, t}}\right)-\mu_{M}\right)^{2}\right) \\
-Z_{B H, t}\left(\frac{B_{H, t}}{P_{t} Z_{t}}-\mu_{B}\right)^{2}-Z_{B W H, t}\left(\frac{F_{t} B_{W H, t}}{P_{t} Z_{t}}-\mu_{W}\right)^{2}
\end{array}\right)\right) \rightarrow \max _{B, C, L, M, X}, \\
& Y_{f, t}\left(\frac{P_{f, t}}{P_{i, t}}\right)^{\theta_{i}}=\omega_{C, i}\left(\frac{P_{i, t}}{P_{C, t}}\right)^{-Z_{\theta C, t}} C_{t}+\omega_{G, i}\left(\frac{P_{i, t}}{P_{G, t}}\right)^{-Z_{\theta G, t}} G_{t}+\omega_{I, i}\left(\frac{P_{i, t}}{P_{I, t}}\right)^{-Z_{\theta I, t}} I_{t} \\
& +\omega_{W 1, i}\left(\frac{P_{i, t}}{F_{t} P_{W 1, t}}\right)^{-Z_{\theta W 1, t}} Y_{W 1, t}+\omega_{W 2, i}\left(\frac{P_{i, t}}{F_{t} P_{W 2, t}}\right)^{-Z_{\theta W 2, t}} Y_{W 2, t}+\sum_{j=1}^{5} \omega_{Q, j, i}\left(\frac{P_{i, t}}{P_{Q, j, t}}\right)^{-Z_{\theta Q, j, t}} Q_{j, t} \\
& D_{f, t}+P_{I, t} I_{f, t}+P_{Q, i, t} Q_{f, t}+W_{t} L_{f, t}+B_{F, f, t}+F_{t} B_{W F, f, t}= \\
& =\left(1-\tau_{Y, i, t}\right) P_{f, t} Y_{f, t}+R_{H, t-1} B_{F, f, t-1}+F_{t} R_{W, t-1} B_{W F, f, t-1}+F_{t} Z_{D W, i, t} \text {, } \\
& Y_{f, t}=Z_{Y F, i, t}\left(Z_{t} L_{f, t}\right)^{1-\alpha_{K F, i}-\alpha_{Q F, i}}\left(K_{f, t-1}\right)^{\alpha_{K F, i}}\left(Q_{f, t}\right)^{\alpha_{Q F, i}} \\
& Y_{f, t}=\left(Z_{Y F, 1, t} Z_{t}\right)^{1-\alpha_{L F, i}-\alpha_{K F, i}-\alpha_{Q F, i}}\left(Z_{t} L_{f, t}\right)^{\alpha_{L F, i}}\left(K_{f, t-1}\right)^{\alpha_{K F, i}}\left(Q_{f, t}\right)^{\alpha_{Q F, i}}, \\
& K_{f, t}=\left(1-\delta_{i}\right) K_{f, t-1}+I_{f, t} \exp \left(-Z_{I, i, t}\left(I_{f, t} / I_{f, t-1}\right)^{2}\right),
\end{aligned}
$$

где $D_{f, t}$ - дивиденды фирмы $f$ в периоде $t$; $Y_{f, t}$ - выпуск фирмы $f$ в периоде $t ; P_{f, t}-$ цена товаров фирмы $f$ в периоде $t$; $I_{f, t}-$ спрос на инвестиционные товары со стороны фирмы $f$ в периоде $t$; $Q_{f, t}-$ спрос на корзину промежуточных товаров со стороны фирмы $f$ в периоде $t ; B_{F, f t}-$ стоимость облигаций в национальной валюте, приобретенных фирмой $f$ в периоде $t ; B_{W F, f t}-$ стоимость облигаций в иностранной валюте, приобретенных фирмой $f$ в периоде $t$; $K_{f, t}-$ объем капитала фирмы $f$ в периоде $t$ и $L_{f, t}-$ объем труда, используемый фирмой $f$ в периоде $t$. Фирма $f$ принадлежит сектору $i$.

Фирмы максимизируют ожидаемую дисконтированную целевую функцию (3) с ограничениями. Целевая функция состоит из дисконтированного потока дивидендов и нескольких негибкостей: негибкость позиции на долговом рынке (в национальной и иностранной валютах), негибкость ценообразования и негибкость фонда оплаты труда. Фирмы действуют на рынке монополистической конкуренции, соответственно они сталкиваются с ограничением на спрос (4). В большинстве ДСОЭР-моделей рынок монополистической конкуренции формируется из единой агрегирующей CES-функции, однако в данной модели для каждого сектора и источника спроса используется собственная агрегирующая CES-функция. Бюджетное ограничение (5) описывает расходы и доходы фирм. Производственная функция (6) - это функция Коба-Дугласа с тремя факторами производства: трудом, капиталом и корзиной промежуточных товаров. Добывающий сектор от- 
личается формой производственной функции (6а), которая отражает зависимость производства от дополнительного экзогенного фактора производства (объем разведанных месторождений) и создает убывающую отдачу от масштаба по управляемым фирмой переменным. Ограничение на эволюцию капитала (7) содержит негибкость инвестиций. Как упоминалось выше, в модели присутствуют 5 секторов.

\section{3. Государство, внешний сектор и балансовые уравнения}

Государство действует в соответствии с бюджетным ограничением и экзогенными правилами политики. Бюджетное ограничение государства выражается следующим образом:

$$
P_{G, t} G_{t}+T_{T R, t}+B_{G, t}=\tau_{L, t} W_{t} L_{t}+\sum_{i=1}^{5} \tau_{Y, i, t} P_{i, t} Y_{i, t}+R_{t-1} B_{G, t-1}+\left(M_{t}-M_{t-1}\right) .
$$

Правило денежной политики:

$$
\ln \left(R_{H, t}\right)=\gamma_{R} \ln \left(R_{H, t-1}\right)+\left(1-\gamma_{R}\right)\left(\begin{array}{l}
\gamma_{R P}\left(\ln \left(\frac{P_{t}}{P_{t-1}}\right)-\bar{p}\right)+\gamma_{R Y}\left(\ln \left(\frac{Y_{D, t}}{Y_{D, t-1}}\right)-\bar{y}\right)+Z_{R, t}+ \\
\gamma_{R F}\left(\ln \left(\frac{F_{t} P_{W, t}}{P_{t}}\right)-\bar{f}\right)+\gamma_{R D F}\left(\ln \left(\frac{F_{t}}{F_{t-1}}\right)-\bar{p}+\overline{p_{W}}\right)
\end{array}\right)
$$

Правила фискальной политики:

$$
\begin{gathered}
\ln \left(\frac{G_{t}}{Y_{D, t}}\right)=\gamma_{G} \ln \left(\frac{G_{t-1}}{Y_{D, t-1}}\right)+\left(1-\gamma_{G}\right)\left(\begin{array}{l}
Z_{G, t}+\gamma_{G B}\left(\frac{B_{G, t}}{P_{t} Y_{D, t}}-\overline{b_{G} e^{-y_{D}}}\right)+ \\
+\gamma_{G Y}\left(\ln \left(\frac{Y_{D, t}}{Y_{D, t-1}}\right)-\bar{y}\right)
\end{array}\right) ; \quad(10) \\
\ln \left(\frac{T_{T R, t}}{Y_{D, t}}\right)=\gamma_{T R} \ln \left(\frac{T_{T R, t-1}}{Y_{D, t-1}}\right)+\left(1-\gamma_{T R}\right)\left(\begin{array}{l}
\left.Z_{T R, t}+\gamma_{T R B}\left(\frac{B_{G, t}}{P_{t} Y_{D, t}}-\overline{b_{G} e^{-y_{D}}}\right)+\right) \\
+\gamma_{T R Y}\left(\ln \left(\frac{Y_{D, t}}{Y_{D, t-1}}\right)-\bar{y}\right)
\end{array}\right) ; \quad \text { (11) } \\
\tau_{L, t}=\gamma_{T L} \tau_{L, t-1}+\left(1-\gamma_{T L}\right)\left(\gamma_{T L B}\left(\frac{B_{G, t}}{P_{t} Y_{D, t}}-\overline{b_{G} e^{-y_{D}}}\right)+\gamma_{T L Y}\left(\ln \left(\frac{Y_{D, t}}{Y_{D, t-1}}\right)-\bar{y}\right)+Z_{T L, t}\right) ; \quad(12) \\
\tau_{Y, i, t}=\gamma_{T Y, i} \tau_{Y, i, t-1}+\left(1-\gamma_{T Y, i}\right)\left(\gamma_{T Y, i B}\left(\frac{B_{G, t}}{P_{t} Y_{D, t}}-\overline{b_{G} e^{-y_{D}}}\right)+\gamma_{T Y, i Y}\left(\ln \left(\frac{Y_{D, t}}{Y_{D, t-1}}\right)-\bar{y}\right)+Z_{T Y, i, t}\right) .
\end{gathered}
$$

Внешний сектор описывается при помощи экзогенных правил и бюджетного ограничения: 


$$
\begin{aligned}
& I M_{t}=\omega_{C, W}\left(\frac{F_{t} P_{W 0, t}}{P_{C, t}}\right)^{-Z_{\theta C, t}} C_{t}+\omega_{G, W}\left(\frac{P_{i, t}}{P_{G, t}}\right)^{-Z_{\theta G, t}} G_{t}+ \\
& +\omega_{I, W}\left(\frac{P_{i, t}}{P_{I, t}}\right)^{-Z_{\theta l, t}} I_{t}+\sum_{j=1}^{5} \omega_{Q, j, W}\left(\frac{P_{i, t}}{P_{Q, j, t}}\right)^{-Z_{\theta Q, j, t}} Q_{j, t} . \\
& E X R_{t}=\sum_{i=1}^{5} \omega_{W 1, i}\left(\frac{P_{i, t}}{F_{t} P_{W 1, t}}\right)^{-Z_{\theta W 1, t}} Y_{W 1, t}+\sum_{i=1}^{5} \omega_{W 2, i}\left(\frac{P_{i, t}}{F_{t} P_{W 2, t}}\right)^{-Z_{\theta W 2, t}} Y_{W 2, t} . \\
& \operatorname{EXN}_{t}=\sum_{i=1}^{5} \omega_{W 1, i}\left(\frac{P_{i, t}}{F_{t} P_{W 1, t}}\right)^{-Z_{\theta W 1, t}} Y_{W 1, t} P_{i, t}+\sum_{i=1}^{5} \omega_{W 2, i}\left(\frac{P_{i, t}}{F_{t} P_{W 2, t}}\right)^{-Z_{\theta W 2, t}} Y_{W 2, t} P_{i, t} . \\
& \left(\frac{Y_{W 1, t}}{Z_{t}}\right)=\gamma_{Y W 1} \ln \left(\frac{Y_{W 1, t-1}}{Z_{t-1}}\right)+\left(1-\gamma_{Y W 1}\right)\left(\gamma_{Y W 1 B}\left(\frac{B_{W, t}}{P_{t} Z_{t}}-\overline{b_{W}}\right)+Z_{Y W 1, t}\right) . \\
& E X N_{t}+\sum_{i=1}^{5} F_{t} Z_{D W, i, t}+\sum_{i=1}^{5} X_{i, W, t} S_{i, t}+\sum_{i=1}^{5} F_{t} R_{W, t-1} B_{W F, i, t-1}+F_{t} R_{W, t-1} B_{W H, i, t-1}+B_{W, t}= \\
& =I M_{t} P_{W 0, t}+\sum_{i=1}^{5} X_{i, W, t-1}\left(S_{i, t}+D_{i, t}\right)+\sum_{i=1}^{5} F_{t} B_{W F, i, t}+F_{t} B_{W H, i, t}+R_{H, t-1} B_{W, t-1} . \\
& \left(\frac{Y_{W 2, t}}{Z_{t}}\right)=\gamma_{Y W 2} \ln \left(\frac{Y_{W 2, t-1}}{Z_{t-1}}\right)+\left(1-\gamma_{Y W 2}\right)\left(\gamma_{Y W 2 B}\left(\frac{B_{W, t}}{P_{t} Z_{t}}-\overline{b_{W}}\right)+Z_{Y W 2, t}\right) \text {. } \\
& \left(\frac{P_{W, t}}{P_{W, t-1}}\right)=\gamma_{P W} \ln \left(\frac{P_{W, t-1}}{P_{W, t-2}}\right)+\left(1-\gamma_{P W}\right)\left(\begin{array}{l}
\gamma_{P W Y W 1}\left(\ln \left(\frac{Y_{W 1, t}}{Z_{t}}\right)-\overline{y_{W 1}}\right)+ \\
+\gamma_{P W Y W 2}\left(\ln \left(\frac{Y_{W 2, t}}{Z_{t}}\right)-\overline{y_{W 2}}\right)+Z_{P W, t}
\end{array}\right) . \\
& \left(\frac{P_{W 0, t}}{P_{W, t}}\right)=\gamma_{P W 0} \ln \left(\frac{P_{W 0, t-1}}{P_{W, t-1}}\right)+\left(1-\gamma_{P W 0}\right)\left(\begin{array}{l}
\gamma_{P W 0 Y W 1}\left(\ln \left(\frac{Y_{W 1, t}}{Z_{t}}\right)-\overline{y_{W 1}}\right)+ \\
+\gamma_{P W 0 Y W 2}\left(\ln \left(\frac{Y_{W 2, t}}{Z_{t}}\right)-\overline{y_{W 2}}\right)+Z_{P W 0, t}
\end{array}\right) . \\
& \left(\frac{P_{W W 1, t}}{P_{W, t}}\right)=\gamma_{P W 1} \ln \left(\frac{P_{W W 1, t-1}}{P_{W, t-1}}\right)+\left(1-\gamma_{P W 1}\right)\left(\begin{array}{l}
\gamma_{P W 1 Y W 1}\left(\ln \left(\frac{Y_{W 1, t}}{Z_{t}}\right)-\overline{y_{W 1}}\right)+ \\
+\gamma_{P W 1 Y W 2}\left(\ln \left(\frac{Y_{W 2, t}}{Z_{t}}\right)-\overline{y_{W 2}}\right)+Z_{P W 1, t}
\end{array}\right) .
\end{aligned}
$$




$$
\begin{gathered}
\left(\frac{P_{W W 2, t}}{P_{W, t}}\right)=\gamma_{P W 2} \ln \left(\frac{P_{W W 2, t-1}}{P_{W, t-1}}\right)+\left(1-\gamma_{P W 2}\right)\left(\begin{array}{l}
\gamma_{P W 2 Y W 1}\left(\ln \left(\frac{Y_{W 1, t}}{Z_{t}}\right)-\overline{y_{W 1}}\right)+ \\
\left.+\gamma_{P W 2 Y W 2}\left(\ln \left(\frac{Y_{W 2, t}}{Z_{t}}\right)-\overline{y_{W 2}}\right)+Z_{P W 2, t}\right)
\end{array}\right) \cdot(23) \\
\ln \left(R_{W, t}\right)=\gamma_{R W} \ln \left(R_{W, t-1}\right)+\left(1-\gamma_{R W}\right)\left(\begin{array}{l}
\gamma_{R W P}\left(\ln \left(\frac{P_{W, t}}{P_{W, t-1}}\right)-\overline{p_{W}}\right)+Z_{R W, t} \\
+\gamma_{R W Y 1}\left(\ln \left(\frac{Y_{W 1, t}}{Y_{W 1, t}}\right)-\bar{z}\right)+\gamma_{R W Y 2}\left(\ln \left(\frac{Y_{W 2, t}}{Y_{W 2, t}}\right)-\bar{z}\right)
\end{array}\right) \\
X_{i, W, t}=\gamma_{X W, i} X_{i, W, t-1}+\left(1-\gamma_{X W, i}\right)\left(\gamma_{X W, i, B}\left(\frac{B_{W, t}}{P_{t} Z_{t}}-\overline{b_{W}}\right)+Z_{X W, i, t}\right) .
\end{gathered}
$$

Формулы (14)-(16) описывают импорт и экспорт как сумму соответствующих спросов. Хотя реальный экспорт, задаваемый формулой (15), не используется в других уравнениях, ему соответствует наблюдаемая переменная, что заставляет привести данное уравнение. Внешний сектор описывается бюджетным ограничением (17), а также экзогенными правилами (18)-(25).

В модели присутствует ряд балансовых ограничений:

$$
\begin{gathered}
B_{H, t}+\sum_{i=1}^{5} B_{F, i, t}+B_{G, t}+B_{W, t}=0 . \\
X_{i, W, t}+X_{i, H, t}=1 . \\
Y_{D, t}=C_{t}+I_{D, t}+G_{t}+E X R_{t}-I M_{t} . \\
I_{D, t}=\sum_{i=1}^{5} I_{i, t} \cdot \\
P_{C, t}{ }^{1-Z_{\theta C, t}}=\omega_{C, W}\left(\frac{F_{t} P_{W 0, t}}{P_{W, t}}\right)^{1-Z_{\theta C, t}}+\sum_{i=1}^{5} \omega_{C, i}\left(P_{i, t}\right)^{1-Z_{\theta C, t} .} \\
P_{G, t}{ }^{1-Z_{\theta G, t}}=\omega_{G, W} L_{i, t} \cdot \\
\left(\frac{F_{t} P_{W 0, t}}{P_{W, t}}\right)^{1-Z_{\theta G, t}}+\sum_{i=1}^{5} \omega_{G, i}\left(P_{i, t}\right)^{1-Z_{\theta G, t} .} \\
P_{I, t}{ }^{1-Z_{\theta \theta, t}}=\omega_{I, W}\left(\frac{F_{t} P_{W 0, t}}{P_{W, t}}\right)^{1-Z_{\theta l, t}}+\sum_{i=1}^{5} \omega_{I, i}\left(P_{i, t}\right)^{1-Z_{\theta I, t}} .
\end{gathered}
$$




$$
\begin{aligned}
P_{Q, j, t}{ }^{1-Z_{\theta Q, j, t}}=\omega_{Q, j, W}\left(\frac{F_{t} P_{W 0, t}}{P_{W, t}}\right)^{1-Z_{\theta Q, j, t}}+\sum_{i=1}^{5} \omega_{Q, j, i}\left(P_{i, t}\right)^{1-Z_{\theta Q, j, t}} . \\
P_{W 1, t}{ }^{1-Z_{\theta W 1, t}}=\omega_{W 1, W}\left(\frac{F_{t} P_{W W 1, t}}{P_{W, t}}\right)^{1-Z_{\theta W 1, t}}+\sum_{i=1}^{5} \omega_{W 1, i}\left(P_{i, t}\right)^{1-Z_{\theta W 1, t}} . \\
P_{W 2, t}{ }^{1-Z_{\theta W 2, t}}=\omega_{W 2, W}\left(\frac{F_{t} P_{W W 2, t}}{P_{W, t}}\right)^{1-Z_{\theta W 2, t}}+\sum_{i=1}^{5} \omega_{W 2, i}\left(P_{i, t}\right)^{1-Z_{\theta W 2, t}} . \\
H_{t}=h_{h} H_{t-1}+C_{t} .
\end{aligned}
$$

Балансовые ограничения отражают следующие естественные особенности экономики: каждая облигация приобретена одним из агентов (26); количество акций фирм сектора $i$ равно единице (27); совокупный спрос состоит из потребления, инвестиций, государственного потребления и чистого экспорта (29); инвестиционный спрос состоит из спроса со стороны каждого из секторов (29); аналогично в отношении спроса на труд (30); уровень цен, соответствующий каждому из источников спроса, является функцией от цен на отдельные товары (31)-(36). Формула (37) описывает механизм формирования привычного уровня потребления.

Bсе экзогенные процессы описываются процессом AR (1) со следующей параметризацией:

$$
z_{\star, t}=\eta_{1,{ }^{*}, t} z_{\star, t-1}+\left(1-\eta_{1,{ }^{*}, t}\right)\left(\eta_{0,{ }^{*}, t}+\varepsilon_{*, t}\right) .
$$

Знак «*» обозначает различные символы в переменных, соответствующих экзогенным процессам, т.е. каждый экзогенный процесс (их названия начинаются с символа $z$ ) описывается при помощи двух параметров - 7 и экзогенного шока $\varepsilon$ (имеющего нормальное распределение) - с остальными аналогичными символами в их обозначениях.

\section{2. Оценка параметров и качество прогнозов}

В российской статистике существует разбиение ВВП по отраслям (или, точнее, добавленной стоимости). Однако данное разложение делается в «базовых ценах», которые далеки от рыночных. Проиллюстрируем это несколькими цифрами из последней (за 2006 г.) таблицы «затраты-выпуск». Выпуск сектора «торговля» составляет 7342 133,9 млн руб., а добавленная стоимость - 4729488 млн руб. в «базовых ценах», в то время как совокупный спрос - 413 526,4 млн руб. по рыночным ценам. Выпуск сектора добычи полезных ископаемых равняется 3641 572,7 млн руб., а добавленная стоимость - 2528 668,2 млн руб. в «базовых ценах», в то время как совокупный спрос составил 7091 269,7 млн руб. по рыночным ценам. В связи с подобной ненадежностью данных российской статистики в модель введены ошибки измерения наблюдаемых переменных.

B модели используются следующие ряды наблюдаемых данных: среднее MIBID и MIBOR $\left(o b s_{R}\right)$; индекс «обрабатывающие производства» $\left(o b s_{Y 2}\right)$; индекс «производство и распределение электроэнергии, газа и воды» (obs $\left.s_{3}\right)$; индекс «добыча 
полезных ископаемых» $\left(o b s_{Y 1}\right)$; темп роста номинального ВВП (obs $\left.s_{P Y}\right)$; оплата труда наемных работников как доля ВВП $\left(o b s_{W L}\right)$; номинальные расходы на конечное потребление домохозяйств как доля ВВП (obs $\left.s_{P C}\right)$; номинальные расходы на конечное потребление государства как доля ВВП (obs $\left.s_{P G}\right)$; номинальный экспорт как доля ВВП $\left(o b s_{P E X}\right)$; номинальный импорт как доля ВВП (obs $\left.s_{P I M}\right)$; номинальные инвестиции как доля ВВП (obs $)$; темп роста реального ВВП (obs $\left.s_{Y}\right)$; темп роста реальных расходов на конечное потребление домохозяйств $\left(o b s_{C}\right)$; темп роста реальных расходов на конечное потребление государства $\left(o b s_{G}\right)$; темп роста реального экспорта $\left(o b s_{E X}\right)$; темп роста реального импорта $\left(o b s_{I M}\right)$; темп роста реальных инвестиций $\left(o b s_{I}\right)$; номинальные доходы консолидированного бюджета как доля ВВП $\left(o b s_{I N C}\right)$; номинальные расходы консолидированного бюджета как доля ВВП $\left(o b s_{t r}\right)$; темп роста номинальной добавленной стоимости сектора $1\left(o b s_{d P Y 1}\right)$; темп роста номинальной добавленной стоимости сектора 2 (obs $\left.{ }_{d P Y 2}\right)$; темп роста номинальной добавленной стоимости сектора 3 ( obs $\left.s_{d P Y 3}\right)$; темп роста номинальной добавленной стоимости сектора $4\left(o b s_{d P Y 4}\right)$; темп роста номинальной добавленной стоимости сектора 5 ( obs $\left.s_{d P Y 5}\right)$; темп роста реальной добавленной стоимости сектоpa $1\left(o b s_{d Y 1}\right)$; темп роста реальной добавленной стоимости сектора $2\left(o b s_{d Y 2}\right)$; темп роста реальной добавленной стоимости сектора 3 (obs $d Y 3)$; темп роста реальной добавленной стоимости сектора $4\left(o b s_{d Y 4}\right)$; темп роста реальной добавленной стоимости сектора 5 (obs $\left.s_{\text {Y } 5}\right)$. Используются данные с корректировкой на сезонность за период с 1-го квартала 1999 по 1-й квартал 2014 г. (для части переменных данные доступны за более короткий период).

Процедура оценки параметров выглядит следующим образом: вначале производится переход к стационарным переменным; затем для каждого из агентов определяются условия оптимальности [Canova, 2007]; после этого находится линейная аппроксимация решения системы динамических уравнений с рациональными ожиданиями [Blanchard, Kahn,1980; Schmitt-Grohe, Uribe, 2004]; при помощи фильтра Калмана проводится расчет функции правдоподобия. Максимум функции правдоподобия составляет 2657,32, что немного лучше показателя для AR(1)модели $(2590,615)$.

Для иллюстрации качества описания статистических данных ДСОЭР-моделью были рассчитаны среднеквадратичные ошибки прогнозов (от 1-го до 4-го кварталов) в рамках выборки и вне выборки (табл. 1). Более подробные оценки качества прогнозов для каждой переменной и горизонта прогнозирования представлены в работе [Иващенко, 2015]. Для сравнения приведены аналогичные показатели для моделей $\mathrm{AR}(1)$ и $\operatorname{VAR}(1)$. Прогноз вне выборки рассчитывался за последние 22 квартала. Это означает, что модель оценивалась без данных за последний квартал (до 4 квартала 2013 г.), и строился прогноз, затем без двух кварталов (до 3 кв. 2013 г.) и так далее, вплоть до оценки без двадцати двух кварталов (до 3 кв. 2008 г.).

Качество прогнозов ДСОЭР-модели в рамках выборки оказывается не очень высоким (модель незначительно уступает AR(1) по всем агрегированным показателям), однако вне выборки ситуация меняется и ДСОЭР-модель оказывается лучшей. Показатели VAR(1)-модели в рамках выборки доминируют над остальными моделями за счет существенно большего числа параметров (1305 параметров против 87 y AR(1) и 411 у ДСОЭР), что приводит к крайне слабым результатам вне выборки. Также следует отметить, что преимущество ДСОЭР-модели над AR(1) наи- 
Таблица 1. Среднеквадратические ошибки прогнозов

\begin{tabular}{|l|c|c|c|c|c|c|}
\hline \multirow{2}{*}{$\begin{array}{c}\text { Агрегированные меры качества } \\
\text { прогнозирования 29 рядов } \\
\text { на горизонтах 1-4 кв. }\end{array}$} & \multicolumn{3}{|c|}{ Вне выборки } & \multicolumn{3}{c|}{ В рамках выборки } \\
\cline { 2 - 7 } & DSGE & AR & VAR & DSGE & AR & VAR \\
\hline Среднее & $6.31 \mathrm{E}-02$ & $6.74 \mathrm{E}-02$ & $1.45 \mathrm{E}+05$ & $7.70 \mathrm{E}-02$ & $6.62 \mathrm{E}-02$ & $3.66 \mathrm{E}-02$ \\
\hline Среднеквадратичное & $8.37 \mathrm{E}-02$ & $1.05 \mathrm{E}-01$ & $6.22 \mathrm{E}+05$ & $1.09 \mathrm{E}-01$ & $9.36 \mathrm{E}-02$ & $5.57 \mathrm{E}-02$ \\
\hline Медиана & $4.74 \mathrm{E}-02$ & $4.86 \mathrm{E}-02$ & $3.18 \mathrm{E}+02$ & $4.82 \mathrm{E}-02$ & $4.51 \mathrm{E}-02$ & $2.46 \mathrm{E}-02$ \\
\hline Среднее отношения (СКО/СКО АR) & $1.06 \mathrm{E}+00$ & $1.00 \mathrm{E}+00$ & $2.20 \mathrm{E}+06$ & $1.17 \mathrm{E}+00$ & $1.00 \mathrm{E}+00$ & $5.45 \mathrm{E}-01$ \\
\hline Медиана отношения (СКО/СКО АR) & $9.84 \mathrm{E}-01$ & $1.00 \mathrm{E}+00$ & $8.03 \mathrm{E}+03$ & $1.08 \mathrm{E}+00$ & $1.00 \mathrm{E}+00$ & $5.46 \mathrm{E}-01$ \\
\hline
\end{tabular}

более заметно при краткосрочных прогнозах, в то время для малых ДСОЭР-моделей США и еврозоны характерно наибольшее превосходство при более долгосрочном прогнозировании [del Negro, Schorfheide, 2012]. Еще одной особенностью данной модели является невысокое качество прогнозов процентных ставок. Хотя это характерно для ДСОЭР-моделей как западных стран [Rubaszek, Skrzypczynski, 2008], так и России [Иващенко, 2013], обычно разница оказывается существенно меньше.

\section{3. Последствия изменения политики}

При анализе ДСОЭР-моделей обычно рассматривают функции реакции на импульс, представляющие собой последствия одного шока (отклонения одного экзогенного шока от нуля в один момент времени). Когда расчеты ведутся в рамках линеаризованной модели, эффект от шока является аддитивным, т. е. последствия не зависят от текущего состояния модели. А при рассмотрении изменения параметров производится сравнение функций реакции на импульс.

В настоящей статье будет использован альтернативный подход. С помощью фильтра Калмана рассчитывается ожидаемое состояние модели при условии имеющихся наблюдений. Далее строятся четыре траектории: 1) ожидаемая траектория (прогноз); 2) ожидаемая траектория при условии изменения группы параметров; 3) ожидаемая траектория при условии ряда одномоментных шоков; 4) ожидаемая траектория при условии ряда перманентных шоков.

Отметим одну деталь, связанную с изменением параметров. В правилах поведения государства присутствуют детерминированные равновесия, однако это является удобным способом параметризации правил поведения государства. То есть данные величины при изучении последствий изменения параметров рассматриваются как константы (и перестают быть детерминированным равновесием).

Итак, проанализируем последствия четырех групп изменений параметров, соответствующих ярким событиям 2014 г., которые можно принять за долгосрочные изменения поведения агентов: 1) обвал цен на нефть; 2) санкции, ограничивающие доступ российских фирм к рынку валютных займов; 3) резкий рост процентных ставок и 4) повышение доли государственных расходов и трансфертов в ВВП (наблюдающееся за несколько последних лет).

Сделаем небольшое отступление, содержащее информацию о единицах измерения в моделях. В экономических моделях, в отличие от физических, используются безразмерные величины (индексы). Это связано с активным применением неполиномиальных зависимостей (например, производственной функции Кобба- 
Дугласа (6)), в которых формирование согласованных единиц измерения проблематично. Аналогичная ситуация в представленной модели: переменные являются безразмерными величинами, преимущественно логарифмами отношений, как и наблюдаемые переменные.

Начнем рассмотрение с обвала цен на нефть (а также на другие экспортируемые Россией товары). Были выбраны шоки ( $\varepsilon_{P W 1, t}$ и $\left.\varepsilon_{P W 2, t}\right)$ такого размера, чтобы цены на экспортируемые товары $\left(P_{W W 1, t}\right.$ и $\left.P_{W W 2, t}\right)$ упали на $50 \%$ от ожидаемого уровня первого квартала 2014 г. (в случае перманентного шока цены упали бы и оставались на этом уровне). При рассмотрении изменения параметров были уменьшены примерно на 0.5 ожидаемые значения соответствующих экзогенных процессов $\left(\eta_{0, P W 1} \mathrm{c}-1.193\right.$ до -1.7, a $\eta_{0, P W 2} \mathrm{c} 1.312$ до 0.8). На рис. 2 показаны четыре траектории для трех основных переменных. Более подробное описание последствий шока экспортных цен в разрезе 15 переменных представлено на рис. Al (см. приложение П2).
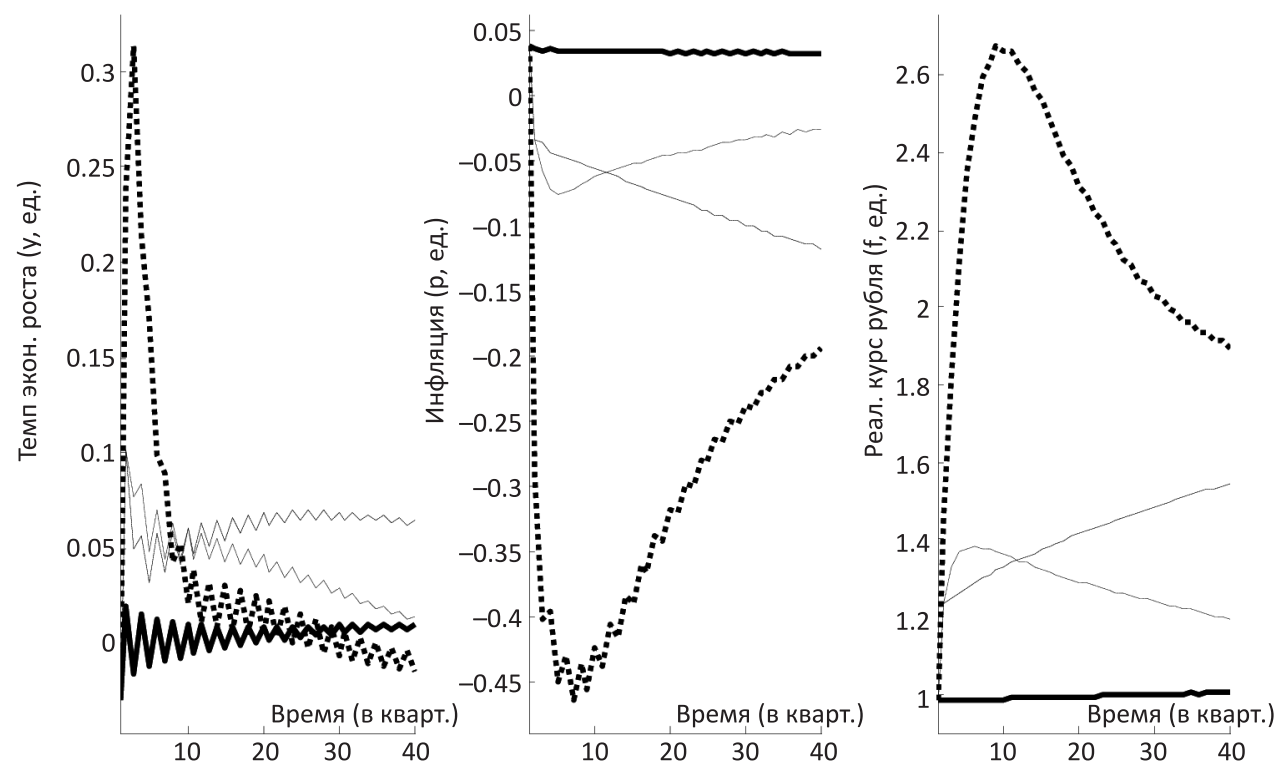

Puc. 2. Последствия 50\%-ного снижения экспортных цен

Обозначения: прогноз ——; изменение параметров . . . . . . .; одномоментный шок .......; перманентный шок - - - - - - .

Очевидно, что снижение экспортных цен способствует экономическому росту. Это является обратной стороной Голландской болезни, механизм которой легко просматривается в данной модели: снижение экспортных цен ведет к снижению экспортной выручки, что способствует ослаблению национальной валюты и опережающему падению импорта, и происходит рост за счет импортозамещения. Увеличение вложения домохозяйств и фирм в иностранную валюту в результате ожидающегося ослабления национальной валюты обеспечивает опережающее ослабление рубля. Денежная политика государства носит противоречивый характер - в ответ на слабость рубля в реальном выражении ставки повышаются («поддержка национальной валюты»), а в ответ на ослабление по отношению к предыдущему периоду ставки снижаются («поддержка банковской системы»). По правилам денежной по- 
литики побеждает «поддержка банковской системы», и ставки падают. Однако это не ведет к инфляции благодаря аккумуляции рублевых ресурсов у государства.

Можно заметить, что реальный рост происходит в добывающем секторе, хотя относительные цены там падают, а также растет пятый сектор (прочее). В то же время торговля и третий сектор (электроэнергия, вода) падают на фоне роста цен. Такова динамика в случае непредсказуемого одномоментного шока и в случае перманентного непредсказуемого шока. Однако при изменении параметров ситуация меняется сильнее, так как из-за перемещения точки детерминированного равновесия происходит сдвиг долгосрочных ожиданий и точки линеаризации, что также влияет на поведение агентов. Для многих величин изменение параметров ведет к тому же знаку последствий, что и шок, но большего масштаба. Однако есть исключения, такие как производство третьего и четвертого секторов или ситуация с доходами и расходами государства. В обоих случаях государство повышает налоги на многие сектора, но при изменении параметров рост налогов оказывается меньше, а изменение структуры экономики больше (причем рост происходит в секторе с низкими налогами - обрабатывающие производства).

Несмотря на сочетание двух свойств данной истории (нетривиальность и правдоподобие), следует отметить одно слабое место использованной методологии. Все расчеты строятся на основе аппроксимации (линейной) поведения модели, полученной при помощи метода возмущений, что означает рост ошибок аппроксимации по мере удаления от детерминированного равновесия (точки линеаризации). Таким образом, так выглядела бы история при небольшом отклонении цен, однако 50\%-ное изменение цен достаточно велико, чтобы нелинейные эффекты могли начать доминировать (в частности, не допустили выхода процентных ставок в отрицательную зону, что произошло в линеаризованной модели).

Теперь рассмотрим последствия санкций, связанные с ограничением доступа фирм России к финансированию в валюте, представленные на рис. 3 и более подробно на рис. А2 (см. приложение). В данной модели это описывается изменением экзогенных процессов, задающих принятый уровень долговой нагрузке в валюте. В рамках изменения параметров средние значения были изменены следующим образом (было: $\eta_{0, B W F 1}=-0.944, \eta_{0, B W F 2}=-1.956, \eta_{0, B W F 3}=-10.869, \eta_{0, B W F 4}=-3.622$, $\eta_{0, B W F 5}=-0.783$; стало: $\eta_{0, B W F 1}=-0.9, \eta_{0, B W F 2}=-1.7, \eta_{0, B W F 3}=-6.0 \mathrm{e}-0, \eta_{0, B W F 4}=-2.5 \mathrm{e}-$ $\left.0, \eta_{0, B W F 5}=-0.7\right)$. То есть секторы, склонные к большим заимствованиям в валюте, сократили свои заимствования (принятый объем заимствований) сильнее. Для анализа последствий шоков $\left(\varepsilon_{W F 1, t} ; \varepsilon_{W F 2, t} ; \varepsilon_{W F 3, t} ; \varepsilon_{W F 4, t} ; \varepsilon_{W F 5, t}\right)$ они были выбраны так, чтобы вектор объема валютных облигаций фирм $\left(B_{W F 1, t} ; B_{W F 2, t} ; B_{W F 3, t} ; B_{W F 4, t}\right.$; $\left.B_{W F 5, t}\right)$ принял значение $(-0.9 ;-1.7 ;-6 ;-2.5 ;-0.7)$, а в случае перманентного шока оставался на этом уровне.

Можно видеть, что сокращение валютных долгов фирм оказывает существенно меньшее воздействие на ключевые показатели по сравнению с шоком экспортных цен. Основное сокращение долговой нагрузки приходится на третий и четвертый секторы, и главные последствия связаны с их реальным выпуском. Однако этот эффект наступает при изменении параметров, а при наличии непредсказуемого одномоментного или перманентного шока отклонения минимальны. Основным последствием шока долговой позиции фирм становится замещение валютных кредитов рублевыми и изменение долговой позиции домохозяйств и государства. 


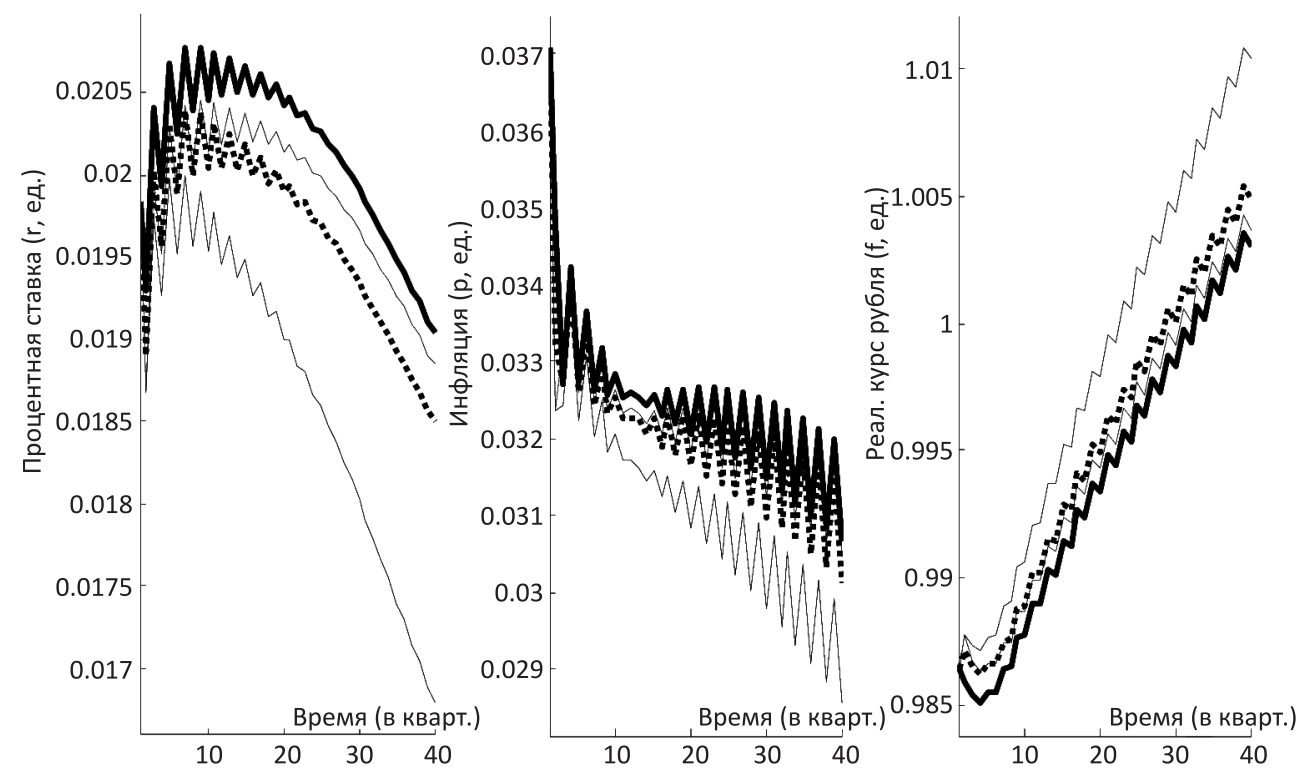

Puc. 3. Последствия ограничения доступа фирм к валютному финансированию

Обозначения: прогноз —; изменение параметров .......; одномоментный шок .......; перманентный шок - - - - - -

Сокращение валютных долгов фирм заставляет их брать дополнительные кредиты в национальной валюте. Фирмы также оказываются заинтересованы в увеличении денежного потока, что стимулирует увеличение выпуска и снижение цен. В результате более низких ставок инфляция немного сокращается и ЦБ снижает ставки. Вследствие более низких ставок все агенты уменьшают вклады в рублевые долговые инструменты (фирмы берут больше рублевых кредитов). Рублевые активы скапливаются на балансе государства, и в рамках антициклической политики оно вынужденно немного поднять налоги. Рост выпуска оказывается минимальным.

Необходимо отметить один значительный факт: существенно большее изменение параметров принятого уровня валютной долговой нагрузки делает модель численно нестабильной. Таким образом, санкции, ограничивающие доступ к валютным займам фирм России, не оказывают заметного влияния, пока не будет достигнут критический масштаб санкций, выводящий систему из динамически стабильного состояния.

Рассмотрим последствия роста процентных ставок на 2\% (подчеркнем, что это составляет 8\% годовых и соответствует фактическому изменению ставок в четвертом квартале 2014 г.), представленные на рис. 4 и более подробно на рис. А3 (см. приложение П2). Отметим, что перманентный шок денежной политики ведет к взрывной траектории, т.е. при непредсказуемом удержании процентных ставок вопреки правилам денежной политики экономика становится крайне нестабильной.

Ситуация с денежной политикой демонстрирует разницу между изменением параметра правил поведения государства и одномоментным непредсказуемым шоком. Если 2\%-ный шок денежной политики ведет к снижению инфляции на 5-6\% 

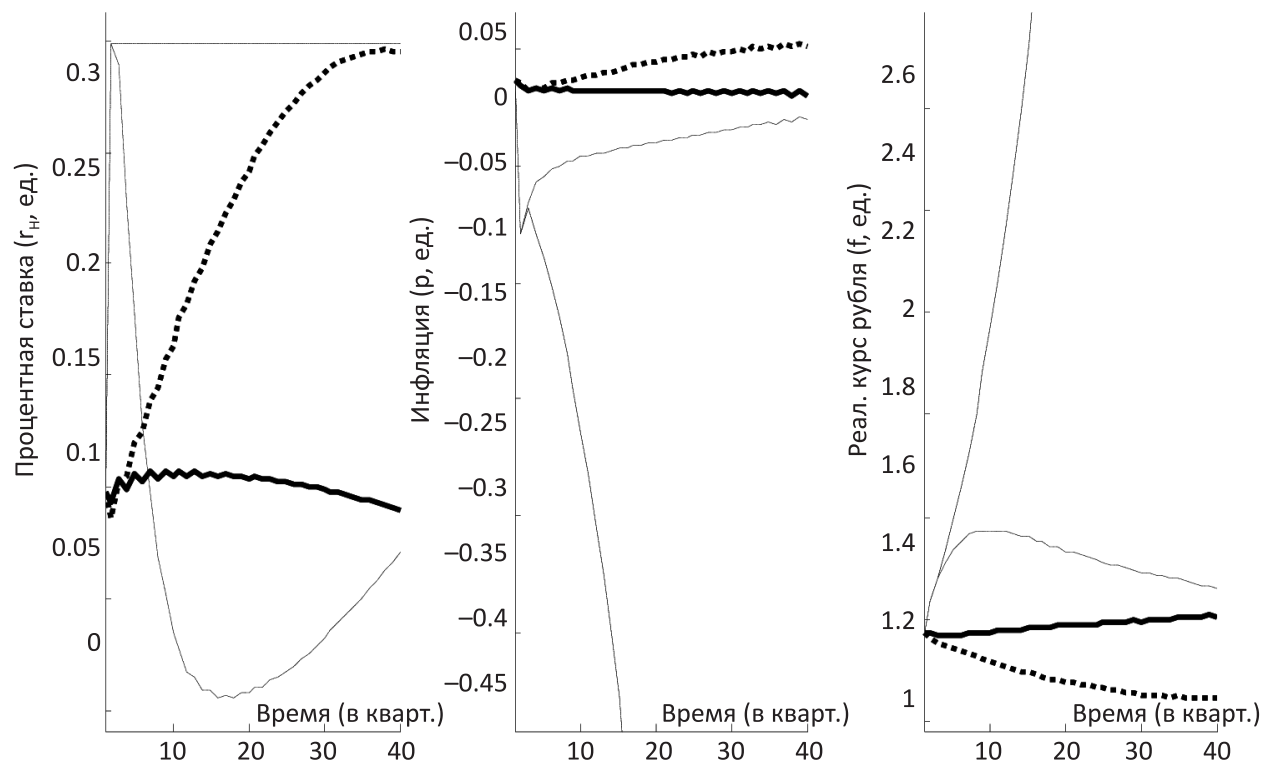

Puc. 4. Последствия роста ставок на $2 \%$

Обозначения: прогноз ——; изменение параметров ......; одномоментный шок ......; перманентный шок - - - - - -

в первые пару кварталов, в то время как аналогичное изменение правил политики ведет к 2\%-ному росту инфляции в долгосрочном периоде и не оказывает краткосрочного воздействия. Шок денежной политики способствует падению темпов роста в первый момент и увеличению в последующие кварталы, в то время как изменение параметров вызывает рост в момент шока и падение в последующие кварталы (причем масштабы меньше, чем у шока). Последствия для темпов роста производства противоположны и в большинстве секторов. Таким образом, для денежной политики фактор предсказуемости поведения носит критический характер.

Изменение параметров денежной политики обеспечивает снижение темпов роста всех секторов, в то время как шок дает краткосрочный позитивный эффект первого (добыча полезных ископаемых) и пятого (остальное) секторов и смешанный для второго (обрабатывающие производства). Разница в поведении начинается с различий в траекториях процентных ставок. Межвременные предпочтения фирм заставляют их существенно увеличить дивиденды в начальный момент времени. Большая часть потока дивидендов направляется во внешний сектор, что ведет к ослаблению национальной валюты. Это означает создание дополнительного спроса для секторов, завязанных на экспорт, а также конкурирующих с импортом. В этот список не попадают третий (электричество, вода, газ) и четвертый (торговля, транспорт, связь) секторы.

Рост процентных ставок ведет к увеличению сбережений и снижению инфляции. Затем домохозяйства соглашаются работать за меньшую заработную плату, сокращают вложения в валютные облигации и объем хранимых денег. В результате отрицательного эмиссионного дохода долговая позиция государства ухудшается, и оно поднимает налоги. Для второго сектора этот эффект пересиливает дополни- 
тельный спрос в исходный момент. Однако после начала снижения ставок долговая позиция государства станет исправляться (за счет больших налогов и эмиссионного дохода), ставки налогов постепенно возвращаются, а позитивный эффект ослабления национальной валюты сохраняется. В конечном итоге второй сектор выходит в плюс.

Теперь рассмотрим последствия роста государственного потребления и трансфертов на 2\% ВВП (всего 4\% ВВП), представленные на рис. 5 и более подробно на рис. А4 (см. приложение). Очевидно, что подобное действие ведет в основном к дополнительным колебаниям темпов роста (суммарный эффект за 40 кварталов менее 0,5\% ВВП). Есть эффекты по изменению отраслевой структуры экономики, но и они невелики (суммарный эффект на реальный выпуск менее 4\%). Перманентный шок ведет к несколько большим колебаниям, но это не меняет ситуацию с малым эффектом увеличения расходов консолидированного бюджета.

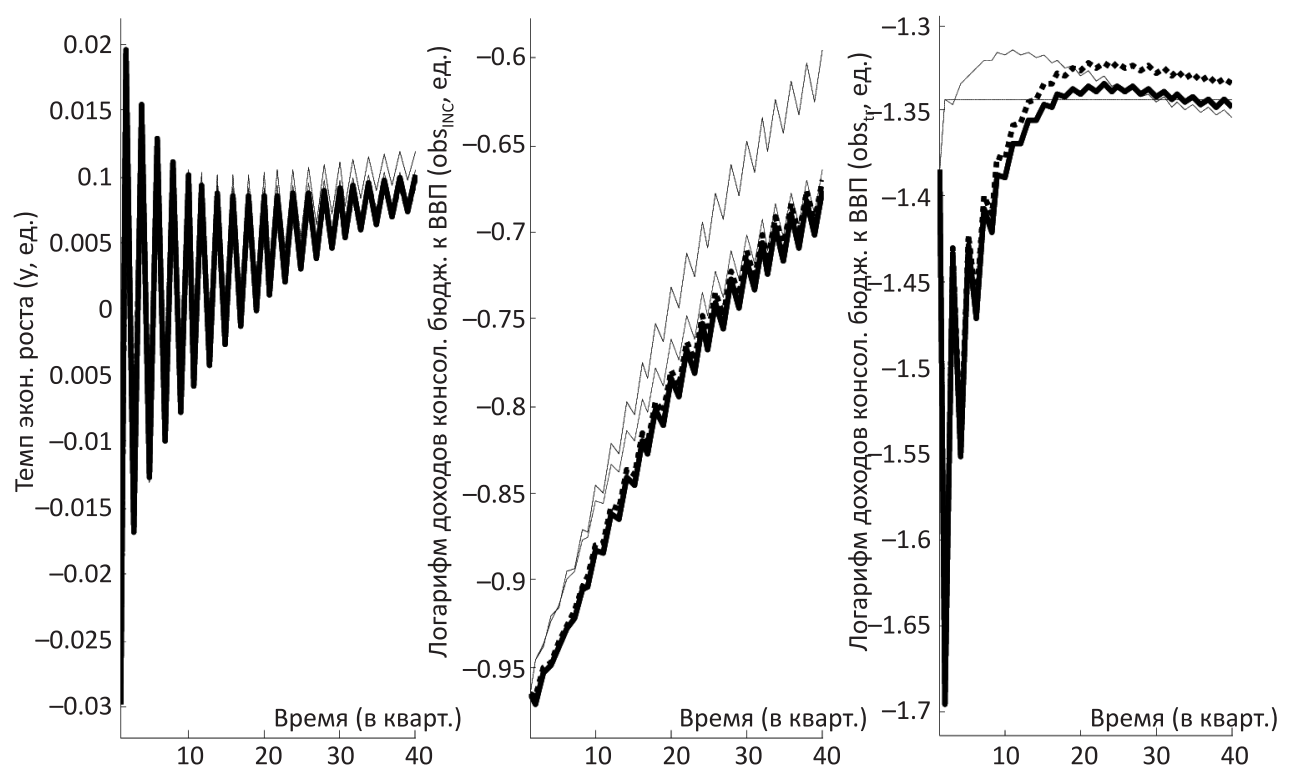

Puc. 5. Последствия повышения государственных расходов и трансфертов

Обозначения: прогноз ——; изменение параметров .......; одномоментный шок ...... . перманентный шок - - - - - - .

Третий (электроэнергия, газ, вода) и четвертый (торговля, транспорт, связь) секторы имеют пониженные темпы роста в случае роста параметров, отвечающих за госрасходы. Это происходит на фоне роста их относительных цен. Налоги на третий и четвертый сектор сильнее остальных реагируют на изменения темпов роста и государственного долга. Соответственно рост расходов государства ведет к опережающему росту налогов на эти секторы.

В случае перманентного шока только четвертый сектор получает монотонный эффект, причем увеличенных темпов роста и относительных цен. В условиях шока доля расходов консолидированного бюджета в ВВП растет быстрее. Госрасходы приходятся в основном на пятый сектор (остальное), и в нем происходит меньшее 
снижение, чем прогнозировалось. Вдобавок спрос со стороны государства слабо эластичен (по сравнению с другими секторами), т. е. прибыль фирм пятого сектора растет. Ожидая возвращения спроса со стороны государства к естественному уровню, фирмы отправляют средства в дивиденды. Однако повышение госрасходов ведет к росту налогов, что стимулирует все секторы сокращать инвестиции, заметная доля которых приходится на пятый сектор. Таким образом, рост производства пятого сектора получается небольшим.

Получив дополнительные средства в форме трансфертов от государства и дивидендов, домохозяйства могут меньше работать, сохраняя прежний уровень потребления. В результате цена труда растет, что снижает конкурентоспособность и инвестиции. Таким образом, в начальный момент наблюдается заметное снижение выпуска, и центральный банк повышает процентные ставки, борясь с избыточной денежной массой и возможной инфляцией. Благодаря этим действиям инфляция незначительно сокращается.

Рост ставок ведет к изменению валютной структуры вкладов домохозяйств и фирм (происходит сокращение валютных вкладов и рост валютных долгов). В результате рубль укрепляется, хотя и несильно, что дополнительно провоцирует спад темпов роста в начальный момент.

Четвертый сектор (торговля, транспорт, связь) практически не взаимодействует с инвестиционным спросом и со спросом со стороны внешнего рынка. Поэтому негативные начальные последствия роста государственных расходов затрагивают его в меньшей степени, чем остальные секторы.

Через некоторое время ухудшение долговой позиции государства завершается из-за повышающихся налогов. Спад инвестиций останавливается, а снижение инфляции позволяет опустить процентные ставки. Четвертый сектор, являясь одним из наиболее закредитованных, выигрывает от этого сильнее других. Вдобавок, избегая негативных последствий снижения инвестиционного спроса, он получает преимущества от роста государственного спроса. В итоге именно этот сектор лучше других реагирует на рост государственных расходов.

Рассматривая динамику относительных цен, можно заметить, что именно четвертый сектор характеризуется наибольшей долей труда как фактора производства. Рост заработных плат вынуждает повышать цены в данном секторе сильнее, чем в остальных. Это особенно легко сделать на фоне повышенного спроса.

\section{Заключение}

Разработанная ДСОЭР-модель не только обладает развитой структурой, отражающей основные механизмы функционирования экономики, но и имеет высокое качество прогнозов, что является одной из ключевых характеристик для любой модели. В рамках выборки качество прогнозов близко к прогнозам AR(1)-модели и заметно уступило прогнозам VAR(1)-модели, однако вне выборки модель показала лучшее качество прогнозов, чем альтернативные подходы. В отличие от моделей меньшего масштаба западных стран, преимущество (над авторегрессионными моделями) данной модели в краткосрочном прогнозировании оказывается больше, чем в долгосрочном прогнозировании. Слабой стороной модели следует указать прогнозы процентных ставок. 
В настоящем исследовании были рассчитаны последствия падения экспортных цен, которые продемонстрировали наличие Голландской болезни в России, а также разнонаправленность воздействия данного фактора на разные секторы. Помимо этого, были определены последствия изменения некоторых других параметров модели и экзогенных шоков, отражающих аналогичное изменение ситуации. Различие последствий воздействий непредсказуемого экзогенного шока и изменения параметров, о котором известно всем агентам, оказалось велико. Особенно ярко это различие проявилось при рассмотрении последствий повышения процентных ставок, где направление последствий для многих ключевых переменных определяется типом повышения процентных ставок. Были проанализированы последствия ограничения объема заимствований фирм в иностранной валюте, которые несущественны до определенного масштаба, а после него модель резко становится не стабильной, т.е. экономика выходит из состояния динамического стохастического равновесия. Последствия одной из антикризисных мер по наращиванию доли государственного потребления и трансфертов населению, которая активно применялась в условиях кризиса 2008-2009 гг, в текущей ситуации не оказывает заметного влияния на темпы экономического роста.

\section{Литература}

Дробышевский С., Полбин А. Декомпозиция динамики макроэкономических показателей РФ на основе DSGE-модели // Экономическая политика. 2015. Т. 10, № 2. С. 20-42.

Иващенко С.М. Динамическая стохастическая модель общего экономического равновесия с банковским сектором и эндогенными дефолтами фирм // Журнал Новой экономической ассоциации. 2013. № 3 (19). С. $27-50$.

Иващенко С. М. ДСОЭР-модель России с 5 секторами // Ес-01/15, Препринт Европейского университета в Санкт-Петербурге, факультет экономики, 2015. URL: http://www.eu.spb.ru/images/ec_dep/wp/ Ec-01_15.pdf p.1-25 (дата обращения: 10.10.15).

Adolfson M., Linde J., Villani M. Forecasting Performance of an Open Economy DSGE Model // Econometric Reviews. 2007. Vol. 26 (2-4). P. 289-328.

Blanchard O., Kahn C. M. The Solution of Linear Difference Models under Rational Expectations // Econometrica. 1980. Vol.48, N 5. P. $1305-1311$.

Canova F. Methods for Applied Macroeconomic Research. Princeton: Princeton University Press, 2007. P. 1-512.

Carvalho C., Lee J. W. Sectoral price facts in a sticky-price model // Staff Reports from Federal Reserve Bank of New York, 2011. N 495. P. 1-53.

O'Doherty J., Tol R. An Environmental Input-Output Model for Ireland // The Economic and Social Review, 2007. Vol. 38, issue 2. P. 157-190.

Lee J. W. Heterogeneous Households in a Sticky Price Model // Departmental Working Papers from Rutgers University. Department of Economics. No 2010-01. P. 1-46.

Lombardo G., Ravenna $F$. The size of the tradable and non-tradable sectors: Evidence from input-output tables for 25 countries // Economics Letters, 2012. Vol. 116, issue 3. P. 558-561.

Lucas R.E. Econometric Policy Evaluation: A Critique // Carnegie-Rochester Conference Series on Public Policy. 1976. Vol. 1, issue 1. P. 19-46.

Malakhovskaya O., Minabutdinov A. Are commodity price shocks important? A Bayesian estimation of a DSGE model for Russia // International Journal of Computational Economics and Econometrics. 2014. Vol.4, issue 1/2. P. 148-180.

Negro M. del, Schorfheide F. DSGE Model-Based Forecasting // Staff Reports from Federal Reserve Bank of New York. 2012. N 554. P. 1-95.

Ojeda J., Parra-Polania J., Vargas C. Natural-Resource Booms, Fiscal Rules and Welfare in a Small Open Economy // Borradores de Economia from Banco de la Republica de Colombia, 2014. N 807. P. 1-32.

Rubaszek M., Skrzypczynski P. On the Forecasting Performance of a Small-Scale DSGE Model // International Journal of Forecasting. 2008. Vol. 24, N 3. P. 498-512. 
Rudolf B., Zurlinden M. A compact open economy DSGE model for Switzerland // Economic Studies from Swiss National Bank, N 2014-08. P. 1-64.

Schmitt-Grohe S., Uribe M. Solving dynamic general equilibrium models using a second-order approximation to the policy function // Journal of Economic Dynamics and Control, 2004. Vol.28(4). P.755-775.

Tovar C. DSGE Models and Central Banks // Economics - The Open-Access, Open-Assessment E-Journal, 2009. Vol.3. P.1-31.

Для цитирования: Иващенко С. М. Многосекторная модель динамического стохастического общего экономического равновесия российской экономики // Вестник Санкт-Петербургского университета. Серия 5. Экономика. 2016. Вып. 3. С. 176-202. DOI: 10.21638/11701/spbu05.2016.310.

\section{References}

Drobyshevskii S., Polbin A. Dekompozitsiia dinamiki makroekonomicheskikh pokazatelei RF na osnove DSGE-modeli [Decomposition of the Structural Shocks Contribution to the Russian Macroeconomic Indicators Dynamics on the Basis of the DSGE Model]. Ekonomicheskaia politika [Economic Policy], 2015, vol. 10, no. 2, pp. 20-42. (In Russian)

Ivashchenko S.M. Dinamicheskaia stokhasticheskaia model' obshchego ekonomicheskogo ravnovesiia s bankovskim sektorom i endogennymi defoltami firm [Dynamic Stochastic General Equilibrium Model with Banks and Endogenous Defaults of Firms]. Zhurnal Novoi ekonomicheskoi assotsiatsii [Journal of the New Economic Association], 2013, no. 3 (19), pp. 27-50. (In Russian)

Ivashchenko S. M. DSOER-model' Rossii s 5 sektorami [DSGE model of Russia with 5 industries]. No Ec-01/15, Working Papers from European University at St. Petersburg, Department of Economics, 2015. Available at: http://www.eu.spb.ru/images/ec_dep/wp/Ec-01_15.pdf p.1-25 (accessed: 10.10.15). (In Russian)

Adolfson M., Linde J., Villani M. Forecasting Performance of an Open Economy DSGE Model. Econometric Reviews, 2007, vol. 26 (2-4), pp. 289-328.

Blanchard O., Kahn C. M. The Solution of Linear Difference Models under Rational Expectations. Econometrica, 1980, vol. 48, no. 5, pp. 1305-1311.

Canova F. Methods for Applied Macroeconomic Research. Princeton, Princeton University Press, 2007, pp. 1-512.

Carvalho C., Lee J. W. Sectoral price facts in a sticky-price model. Staff Reports from Federal Reserve Bank of New York, 2011, no. 495, pp.1-53.

O’Doherty J., Tol R. An Environmental Input-Output Model for Ireland. The Economic and Social Review, 2007, vol. 38, issue 2, pp. 157-190.

Lee J.W. Heterogeneous Households in a Sticky Price Model. Departmental Working Papers from Rutgers University. Department of Economics. No 2010-01, pp. 1-46

Lombardo G., Ravenna F. The size of the tradable and non-tradable sectors: Evidence from input-output tables for 25 countries. Economics Letters, 2012, vol. 116, issue 3, pp. 558-561.

Lucas R.E. Econometric Policy Evaluation: A Critique. Carnegie-Rochester Conference Series on Public Policy, 1976, vol. 1, issue 1, pp. 19-46.

Malakhovskaya O., Minabutdinov A. Are commodity price shocks important? A Bayesian estimation of a DSGE model for Russia. International Journal of Computational Economics and Econometrics, 2014, vol. 4, issue $1 / 2$, pp. $148-180$.

Negro M. del, Schorfheide F. DSGE Model-Based Forecasting. Staff Reports from Federal Reserve Bank of New York, 2012, no. 554, pp. 1-95.

Ojeda J., Parra-Polania J., Vargas C. Natural-Resource Booms, Fiscal Rules and Welfare in a Small Open Economy. Borradores de Economia from Banco de la Republica de Colombia, 2014, no. 807, pp. 1-32.

Rubaszek M., Skrzypczynski P. On the Forecasting Performance of a Small-Scale DSGE Model. International Journal of Forecasting, 2008, vol. 24, no. 3, pp.498-512.

Rudolf B., Zurlinden M. A compact open economy DSGE model for Switzerland. Economic Studies from Swiss National Bank, no. 2014-08, pp. 1-64.

Schmitt-Grohe S., Uribe M. Solving dynamic general equilibrium models using a second-order approximation to the policy function. Journal of Economic Dynamics and Control, 2004, vol. 28(4), pp. 755-775.

Tovar C. DSGE Models and Central Banks. Economics - The Open-Access, Open-Assessment E-Journal, 2009, vol. 3, pp. 1-31.

For citation: Ivashchenko S. M. Multiple Sectors DSGE Model of Russia. Vestnik of Saint Petersburg University. Series 5. Economics, 2016, issue 3, pp. 176-202. DOI: 10.21638/11701/spbu05.2016.310. 


\section{Приложение}

Таблица А1. Переменные ДСОЭР-модели

\begin{tabular}{|c|c|c|}
\hline Переменная & Описание & Стационарная переменная \\
\hline $\mathrm{B}_{\mathrm{F}, \mathrm{i}, \mathrm{t}}$ & $\begin{array}{l}\text { Объем облигаций в национальной валюте, приобретен- } \\
\text { ных фирмами сектора } i \text { в периоде } t\end{array}$ & $b_{F, i, t}=B_{F, i, t} / P_{t} Z_{t}$ \\
\hline $\mathrm{B}_{\mathrm{G}, \mathrm{t}}$ & $\begin{array}{l}\text { Объем облигаций в национальной валюте, приобретен- } \\
\text { ных государством в периоде } t\end{array}$ & $b_{G, t}=B$ \\
\hline $\mathrm{B}_{\mathrm{H}, \mathrm{t}}$ & $\begin{array}{l}\text { Объем облигаций в национальной валюте, приобретен- } \\
\text { ных домохозяйствами в периоде } t\end{array}$ & $b_{H, t}=$ \\
\hline $\mathrm{B}_{\mathrm{W}, \mathrm{t}}$ & $\begin{array}{l}\text { Объем облигаций в национальной валюте, приобретен- } \\
\text { ных внешним сектором в периоде } t\end{array}$ & $b_{W, t}=$ \\
\hline $\mathrm{B}_{\mathrm{WF}, \mathrm{i}, \mathrm{t}}$ & $\begin{array}{l}\text { Объем облигаций в иностранной валюте, приобретен- } \\
\text { ных фирмами сектора } i \text { в периоде } t\end{array}$ & $b_{W F, i, t}$ \\
\hline $\mathrm{B}_{\mathrm{WH}, \mathrm{t}}$ & $\begin{array}{l}\text { Объем облигаций в иностранной валюте, приобретен- } \\
\text { ных домохозяйствами в периоде } t\end{array}$ & $b_{W H, t}=$ \\
\hline $\mathrm{C}_{\mathrm{t}}$ & Потребление домохозяйств в периоде $t$ & $c_{t}=\ln ($ \\
\hline $\mathrm{D}_{\mathrm{i}, \mathrm{t}}$ & Дивиденды фирм сектора $i$ в периоде $t$ & $d_{i, t}=D$ \\
\hline $\mathrm{G}_{\mathrm{t}}$ & Государственное потребление в периоде $t$ & $g_{t}=\ln \left(G_{t} / Z_{t}\right)$ \\
\hline $\mathrm{F}_{\mathrm{t}}$ & $\begin{array}{l}\text { Обменный курс (число единиц нац. валюты за единицу } \\
\text { ин. валюты) в периоде } t\end{array}$ & $f_{t}=\ln \left(\frac{F_{t} P_{W, t}}{P_{t}}\right.$ \\
\hline $\mathrm{H}_{\mathrm{t}}$ & Привычный уровень потребления в периоде $t$ & $h_{t}=\ln \left(H_{t} / Z_{t}\right)$ \\
\hline $\mathrm{I}_{\mathrm{D}, \mathrm{t}}$ & Суммарные инвестиции всех секторов в периоде $t$ & $i_{D, t}=\ln \left(I_{D, t} / Z_{t}\right)$ \\
\hline $\mathrm{I}_{\mathrm{F}, \mathrm{i}, \mathrm{t}}$ & Инвестиции фирм сектора $i$ в периоде $t$ & $i_{F, i, t}=\ln \left(I_{F, i, t} / Z_{t}\right)$ \\
\hline $\mathrm{K}_{\mathrm{i}, \mathrm{t}}$ & Капитал фирм сектора $i$ в периоде $t$ & $k_{i, t}=\ln \left(K_{i, t} / Z_{t}\right)$ \\
\hline $\mathrm{L}_{\mathrm{t}}$ & Предложение труда в периоде $t$ & $l_{t}=\ln \left(L_{t}\right)$ \\
\hline $\mathrm{L}_{\mathrm{F}, \mathrm{i}, \mathrm{t}}$ & Спрос на труд со стороны фирм сектора $i$ в периоде $t$ & $l_{F, i, t}=\ln \left(L_{F, i, t}\right)$ \\
\hline $\mathrm{M}_{\mathrm{t}}$ & Денежная масса в периоде $t$ & $m_{t}=\ln \left(M_{t} / P_{t} Z_{t}\right)$ \\
\hline $\mathrm{EXN}_{\mathrm{t}}$ & Номинальный экспорт в периоде $t$ & $\operatorname{exn}_{t}=\left(E X N_{t} / Z_{t}\right)$ \\
\hline
\end{tabular}




\begin{tabular}{|c|c|c|}
\hline Переменная & Описание & Стационарная переменная \\
\hline $\mathrm{EXR}_{\mathrm{t}}$ & Реальный экспорт в периоде $t$ & $\operatorname{exr}_{t}=\left(E X R_{t} / P_{t} Z_{t}\right)$ \\
\hline $\mathrm{IM}_{\mathrm{t}}$ & Реальный импорт в периоде $t$ & $i m_{t}=\left(I M_{t} / Z_{t}\right)$ \\
\hline $\mathrm{P}_{\mathrm{t}}$ & Уровень цен в периоде $t$ & $p_{t}=\ln \left(P_{t} / P_{t-1}\right)$ \\
\hline $\mathrm{P}_{\mathrm{C}, \mathrm{t}}$ & $\begin{array}{l}\text { Цена корзины товаров, потребляемых домохозяйства- } \\
\text { ми в периоде } t\end{array}$ & $P_{C, t}=\ln \left(P_{C, t} / P_{t}\right)$ \\
\hline $\mathrm{P}_{\mathrm{F}, \mathrm{i}, \mathrm{t}}$ & Цена товаров фирм сектора $i$ в периоде $t$ & $p_{F, i, t}=\ln \left(P_{F, i, t} / P_{t}\right)$ \\
\hline $\mathrm{P}_{\mathrm{G}, \mathrm{t}}$ & $\begin{array}{l}\text { Цена корзины товаров, потребляемых государством } \\
\text { в периоде } t\end{array}$ & $p_{G, t}=\ln \left(P_{G, t} / P_{t}\right)$ \\
\hline $\mathrm{P}_{\mathrm{I}, \mathrm{t}}$ & $\begin{array}{l}\text { Цена корзины товаров, идущих на инвестиции в пери- } \\
\text { оде } t\end{array}$ & $p_{I, t}=\ln \left(P_{I, t} / P_{t}\right)$ \\
\hline $\mathrm{P}_{\mathrm{Q}, \mathrm{i}, \mathrm{t}}$ & $\begin{array}{l}\text { Цена корзины промежуточных товаров, используемых } \\
\text { в производстве фирм сектора } i \text { в периоде } t\end{array}$ & $p_{Q, i, t}=\ln \left(P_{Q, i, t} / P_{t}\right)$ \\
\hline $\mathrm{P}_{\mathrm{W}, \mathrm{t}}$ & Уровень цен на рынке внешнего сектора в периоде $t$ & $p_{W, t}=\ln \left(P_{W, t} / P_{W, t-1}\right)$ \\
\hline $\mathrm{P}_{\mathrm{W} 0, \mathrm{t}}$ & Цена импортных товаров в периоде $t$ & $p_{W 0, t}=\ln \left(P_{W 0, t} / P_{W, t}\right)$ \\
\hline $\mathrm{P}_{\mathrm{W} 1, \mathrm{t}}$ & $\begin{array}{l}\text { Уровень цен в первом секторе экспортных товаров в пе- } \\
\text { риоде } t\end{array}$ & $p_{W 1, t}=\ln \left(P_{W 1, t} / P_{W, t}\right)$ \\
\hline$P_{W W 1, t}$ & $\begin{array}{l}\text { Уровень цен товаров, производимых внешним сектором } \\
\text { для первого сектора экспортных товаров в периоде } t\end{array}$ & $p_{W 1, t}=\ln \left(P_{W 1, t} / P_{W, t}\right)$ \\
\hline $\mathrm{P}_{\mathrm{W} 2, \mathrm{t}}$ & $\begin{array}{l}\text { Уровень цен во втором секторе экспортных товаров } \\
\text { в периоде t }\end{array}$ & $p_{W 2, t}=\ln \left(P_{W 2, t} / P_{W, t}\right)$ \\
\hline $\mathrm{P}_{\mathrm{WW} 2, \mathrm{t}}$ & $\begin{array}{l}\text { Уровень цен товаров, производимых внешним сектором } \\
\text { для второго сектора экспортных товаров в периоде } t\end{array}$ & $p_{W 2, t}=\ln \left(P_{W 2, t} / P_{W, t}\right)$ \\
\hline $\mathrm{Q}_{\mathrm{i}, \mathrm{t}}$ & $\begin{array}{l}\text { Объем промежуточных товаров, используемых в про- } \\
\text { изводстве фирм сектора } i \text { в периоде } t\end{array}$ & $q_{i, t}=\ln \left(Q_{i, t} / Z_{t}\right)$ \\
\hline $\mathrm{R}_{\mathrm{H}, \mathrm{t}}$ & Процентная ставка в национальной валюте периоде $t$ & $r_{H, t}=\ln \left(R_{H, t}\right)$ \\
\hline $\mathrm{R}_{\mathrm{W}, \mathrm{t}}$ & Процентная ставка в иностранной валюте периоде $t$ & $r_{W, t}=\ln \left(R_{W, t}\right)$ \\
\hline$S_{i, t}$ & Цена акций фирм сектора $i$ в периоде $t$ & $s_{i, t}=\ln$ \\
\hline$\tau_{L, t}$ & Ставка налогов на труд в периоде $t$ & $\tau_{L, t}=\tau_{L, t}$ \\
\hline$\tau_{Y, i, t}$ & Ставка налогов на выпуск сектора $i$ в периоде $t$ & $\tau_{Y, i, t}=\tau_{Y, i, t}$ \\
\hline $\mathrm{T}_{\mathrm{TR}, \mathrm{t}}$ & Трансферты от государства в периоде $t$ & $\tau_{T R, t}=\ln \left(T_{T R, t} / P_{t} Z_{t}\right)$ \\
\hline $\mathrm{W}_{\mathrm{t}}$ & Заработная плата в периоде $t$ & $w_{t}=\ln \left(W_{t} / P_{t} Z_{t}\right)$ \\
\hline
\end{tabular}




\begin{tabular}{|c|c|c|}
\hline Переменная & Описание & Стационарная переменная \\
\hline $\mathrm{X}_{\mathrm{i}, \mathrm{H}, \mathrm{t}}$ & $\begin{array}{l}\text { Количество акций фирм сектора } i \text {, приобретенных до- } \\
\text { мохозяйствами в периоде } t\end{array}$ & $x_{i, H, t}=X_{i, H, t}$ \\
\hline $\mathrm{X}_{\mathrm{i}, \mathrm{W}, \mathrm{t}}$ & $\begin{array}{l}\text { Количество акций фирм сектора } i \text {, приобретенных } \\
\text { внешним сектором в периоде } t\end{array}$ & $x_{i, W, t}=X_{i, W, t}$ \\
\hline $\mathrm{Y}_{\mathrm{D}, \mathrm{t}}$ & Совокупный спрос в периоде $t$ & $y_{D, t}=\ln \left(Y_{D, t} / Z_{t}\right)$ \\
\hline$Y_{i, t}$ & Выпуск фирм сектора $i$ в периоде $t$ & $y_{i, t}=\ln \left(Y_{i, t} / Z_{t}\right)$ \\
\hline $\mathrm{Y}_{\mathrm{W} 1, \mathrm{t}}$ & $\begin{array}{l}\text { Объем спроса со стороны первого экспортного сектора } \\
\text { в периоде } t\end{array}$ & $y_{W 1, t}=\ln \left(Y_{W 1, t} / Z_{t}\right)$ \\
\hline $\mathrm{Y}_{\mathrm{W} 2, \mathrm{t}}$ & $\begin{array}{l}\text { Объем спроса со стороны второго экспортного сектора } \\
\text { в периоде } t\end{array}$ & $y_{W 2, t}=\ln \left(Y_{W 2, t} / Z_{t}\right)$ \\
\hline$Z_{\beta, t}$ & $\begin{array}{l}\text { Экзогенный процесс, характеризующий межвременные } \\
\text { предпочтения домохозяйств }\end{array}$ & $z_{\beta, t}=\ln \left(Z_{\beta, t} / Z_{\beta, t-1}\right)$ \\
\hline $\mathrm{Z}_{\mathrm{BF}, \mathrm{i}, \mathrm{t}}$ & $\begin{array}{l}\text { Экзогенный процесс, характеризующий принятый уро- } \\
\text { вень долговой нагрузки в национальной валюте для } \\
\text { фирм сектора } i\end{array}$ & $z_{B F, i, t}=Z_{B F, i, t}$ \\
\hline $\mathrm{Z}_{\mathrm{BH}, \mathrm{t}}$ & $\begin{array}{l}\text { Экзогенный процесс, характеризующий негибкость } \\
\text { долговой позиции в национальной валюте домохо- } \\
\text { зяйств }\end{array}$ & $z_{B H, t}=\ln \left(Z_{B H, t} / Z_{t}^{1-\omega_{C}}\right)$ \\
\hline $\mathrm{Z}_{\mathrm{BW}, \mathrm{t}}$ & $\begin{array}{l}\text { Экзогенный процесс, характеризующий позицию внеш- } \\
\text { него сектора в долговых инструментах в нац. валюте }\end{array}$ & $z_{B W, t}=Z_{B W, t}$ \\
\hline$Z_{\mathrm{BWF}, \mathrm{i}, \mathrm{t}}$ & $\begin{array}{l}\text { Экзогенный процесс, характеризующий принятый } \\
\text { уровень долговой нагрузки в иностранной валюте для } \\
\text { фирм сектора } i\end{array}$ & $z_{B W F, i, t}$ \\
\hline $\mathrm{Z}_{\mathrm{BWH}, \mathrm{t}}$ & $\begin{array}{l}\text { Экзогенный процесс, характеризующий негибкость } \\
\text { долговой позиции в иностранной валюте домохозяйств }\end{array}$ & $z_{B W H, t}=\ln \left(Z_{B W H, t} / Z_{t}^{1-\omega_{C}}\right)$ \\
\hline $\mathrm{Z}_{\mathrm{DW}, \mathrm{i}, \mathrm{t}}$ & $\begin{array}{l}\text { Экзогенный процесс, характеризующий чистый финан- } \\
\text { совый поток из внешнего сектора фирм сектора } i\end{array}$ & $z_{D W, i, t}=\frac{Z_{D W, i, t}}{P_{W, t} Z_{t}}$ \\
\hline $\mathrm{Z}_{\mathrm{FWL}, \mathrm{i}, \mathrm{t}}$ & $\begin{array}{l}\text { Экзогенный процесс, характеризующий негибкость } \\
\text { фонда оплаты труда фирм сектора } i\end{array}$ & $z_{F W L, i, t}=\ln \left(Z_{F W L, i, t}\right)$ \\
\hline $\mathrm{Z}_{\mathrm{G}, \mathrm{t}}$ & $\begin{array}{l}\text { Экзогенный процесс, характеризующий государствен- } \\
\text { ное потребление }\end{array}$ & $z_{G, t}=\ln \left(Z_{G, t}\right)$ \\
\hline $\mathrm{Z}_{\mathrm{I}, \mathrm{i}, \mathrm{t}}$ & $\begin{array}{l}\text { Экзогенный процесс, характеризующий убывающую } \\
\text { эффективность инвестиций в сектора } i\end{array}$ & $z_{I, i, t}=\ln \left(Z_{I, i, t}\right)$ \\
\hline $\mathrm{Z}_{\mathrm{L}, \mathrm{t}}$ & $\begin{array}{l}\text { Экзогенный процесс, характеризующий объем труда } \\
\text { в распоряжении домохозяйств }\end{array}$ & $z_{L, t}=\ln \left(Z_{L, t} / Z_{t}^{1-\omega_{C}}\right)$ \\
\hline $\mathrm{Z}_{\mathrm{M}, \mathrm{t}}$ & $\begin{array}{l}\text { Экзогенный процесс, характеризующий предпочтение } \\
\text { ликвидности }\end{array}$ & $z_{M, t}=\ln \left(Z_{M, t} / Z_{t}^{-\omega_{C}}\right)$ \\
\hline $\mathrm{Z}_{\mathrm{P}, \mathrm{i}, \mathrm{t}}$ & $\begin{array}{l}\text { Экзогенный процесс, характеризующий уровень цено- } \\
\text { вой негибкости в сектора } i\end{array}$ & $z_{P, i, t}=\ln \left(Z_{P, i, t}\right)$ \\
\hline $\mathrm{Z}_{\mathrm{PW}, \mathrm{t}}$ & $\begin{array}{l}\text { Экзогенный процесс, характеризующий уровень ин- } \\
\text { фляции во внешнем секторе }\end{array}$ & $z_{P W, t}=Z_{P W, t}$ \\
\hline $\mathrm{Z}_{\mathrm{PW} 0, \mathrm{t}}$ & $\begin{array}{l}\text { Экзогенный процесс, характеризующий уровень цен на } \\
\text { импортные товары }\end{array}$ & $z_{P W 0, t}=Z_{P W 0, t}$ \\
\hline
\end{tabular}




\begin{tabular}{|c|c|c|}
\hline Переменная & Описание & Стационарная переменная \\
\hline $\mathrm{Z}_{\mathrm{PW} 1, \mathrm{t}}$ & \begin{tabular}{|l|} 
Экзогенный процесс, характеризующий уровень цен то- \\
варов первого сектора экспортных товаров
\end{tabular} & $z_{P W 1, t}=Z_{P W 1, t}$ \\
\hline $\mathrm{Z}_{\mathrm{PW} 2, \mathrm{t}}$ & \begin{tabular}{|l|} 
Экзогенный процесс, характеризующий уровень цен то- \\
варов второго сектора экспортных товаров
\end{tabular} & $z_{P W 2, t}=Z_{P W 2, t}$ \\
\hline $\mathrm{Z}_{\mathrm{QF}, \mathrm{i}, \mathrm{t}}$ & $\begin{array}{l}\text { Экзогенный процесс, характеризующий уровень негиб- } \\
\text { кости использования промежуточных товаров в секто- } \\
\text { ра } i\end{array}$ & $z_{Q F, i, t}=\ln \left(Z_{Q F, i, t}\right)$ \\
\hline $\mathrm{Z}_{\mathrm{R}, \mathrm{t}}$ & $\begin{array}{l}\text { Экзогенный процесс, характеризующий денежную по- } \\
\text { литику }\end{array}$ & $z_{R, t}=Z_{R, t}$ \\
\hline $\mathrm{Z}_{\mathrm{RW}, \mathrm{t}}$ & $\begin{array}{l}\text { Экзогенный процесс, характеризующий денежную по- } \\
\text { литику внешнего сектора }\end{array}$ & $z_{R W, t}=Z_{R W, t}$ \\
\hline$Z_{\theta C, t}$ & $\begin{array}{l}\text { Экзогенный процесс, характеризующий эластичность } \\
\text { спроса домохозяйств }\end{array}$ & $z_{\theta C, t}=Z_{\theta C, t}$ \\
\hline$Z_{\theta G, t}$ & $\begin{array}{l}\text { Экзогенный процесс, характеризующий эластичность } \\
\text { спроса государства }\end{array}$ & $z_{\theta G, t}=Z_{\theta G, t}$ \\
\hline$Z_{\theta I, t}$ & $\begin{array}{l}\text { Экзогенный процесс, характеризующий эластичность } \\
\text { инвестиционного спроса }\end{array}$ & $z_{\theta I, t}=Z_{\theta I, t}$ \\
\hline$Z_{\theta Q, i, t}$ & \begin{tabular}{|l|} 
Экзогенный процесс, характеризующий эластичность \\
спроса на промежуточные товары со стороны сектора $i$
\end{tabular} & $z_{\theta Q, i, t}=Z_{\theta Q, i, t}$ \\
\hline$Z_{\theta W 1, t}$ & $\begin{array}{l}\text { Экзогенный процесс, характеризующий эластичность } \\
\text { спроса первого сектора экспортных товаров }\end{array}$ & $z_{\theta W 1, t}=Z_{\theta W 1, t}$ \\
\hline$Z_{\theta W 2, t}$ & $\begin{array}{l}\text { Экзогенный процесс, характеризующий эластичность } \\
\text { спроса второго сектора экспортных товаров }\end{array}$ & $z_{\theta W 2, t}=Z_{\theta W 2, t}$ \\
\hline $\mathrm{Z}_{\tau \mathrm{L}, \mathrm{t}}$ & Экзогенный процесс, характеризующий налоги на труд & $z_{\tau L, t}=Z_{\tau L, t}$ \\
\hline $\mathrm{Z}_{\tau \mathrm{Y}, \mathrm{i}, \mathrm{t}}$ & $\begin{array}{l}\text { Экзогенный процесс, характеризующий налоги в секто- } \\
\text { ра } i\end{array}$ & $z_{\tau Y, i, t}=Z_{\tau Y, i, t}$ \\
\hline $\mathrm{Z}_{\mathrm{TR}, \mathrm{t}}$ & $\begin{array}{|lll|}\begin{array}{l}\text { Экзогенный } \\
\text { трансфертов }\end{array} & & \\
\end{array}$ & $z_{T R, t}=Z_{T R, t}$ \\
\hline $\mathrm{Z}_{\mathrm{XW}, \mathrm{i}, \mathrm{t}}$ & $\begin{array}{l}\text { Экзогенный процесс, характеризующий позицию внеш- } \\
\text { него сектора в акциях сектора } i\end{array}$ & $z_{X W, i, t}=Z_{X W, i, t}$ \\
\hline $\mathrm{Z}_{\mathrm{YW} 1, \mathrm{t}}$ & $\begin{array}{l}\text { Экзогенный процесс, характеризующий объем спроса } \\
\text { первого сектора экспортных товаров }\end{array}$ & $z_{Y W 1, t}=Z_{Y W 1, t}$ \\
\hline $\mathrm{Z}_{\mathrm{YW} 2, \mathrm{t}}$ & \begin{tabular}{|l|} 
Экзогенный процесс, характеризующий объем спроса \\
второго сектора экспортных товаров
\end{tabular} & $z_{Y W 2, t}=Z_{Y W 2, t}$ \\
\hline$Z_{Y, i, t}$ & $\begin{array}{l}\text { Экзогенный процесс, характеризующий технологиче- } \\
\text { ский прогресс в сектора } i\end{array}$ & $z_{Y, i, t}=\ln \left(Z_{Y, i, t}\right)$ \\
\hline $\mathrm{Z}_{\mathrm{t}}$ & $\begin{array}{l}\text { Экзогенный процесс, характеризующий технологиче- } \\
\text { ский прогресс }\end{array}$ & $z_{t}=\ln \left(Z_{t} / Z_{t-1}\right)$ \\
\hline
\end{tabular}



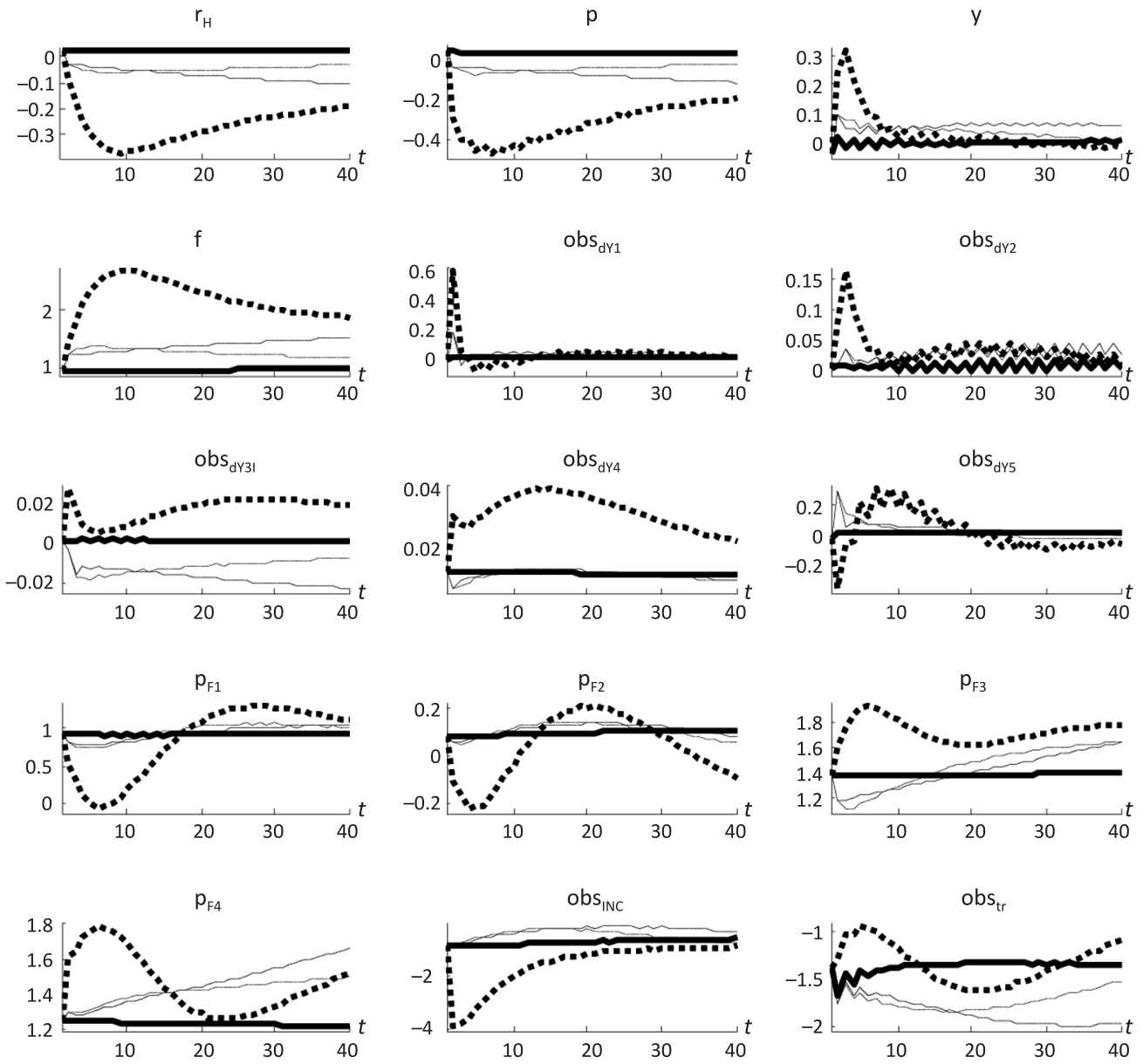

Puc. A1. Последствия 50\%-ного снижения экспортных цен Вертикальная ось: значение переменной. Горизонтальная ось: время в кварталах.

Обозначения: прогноз ——; изменение параметров .......; одномоментный шок . . . . . . перманентный шок -------. 

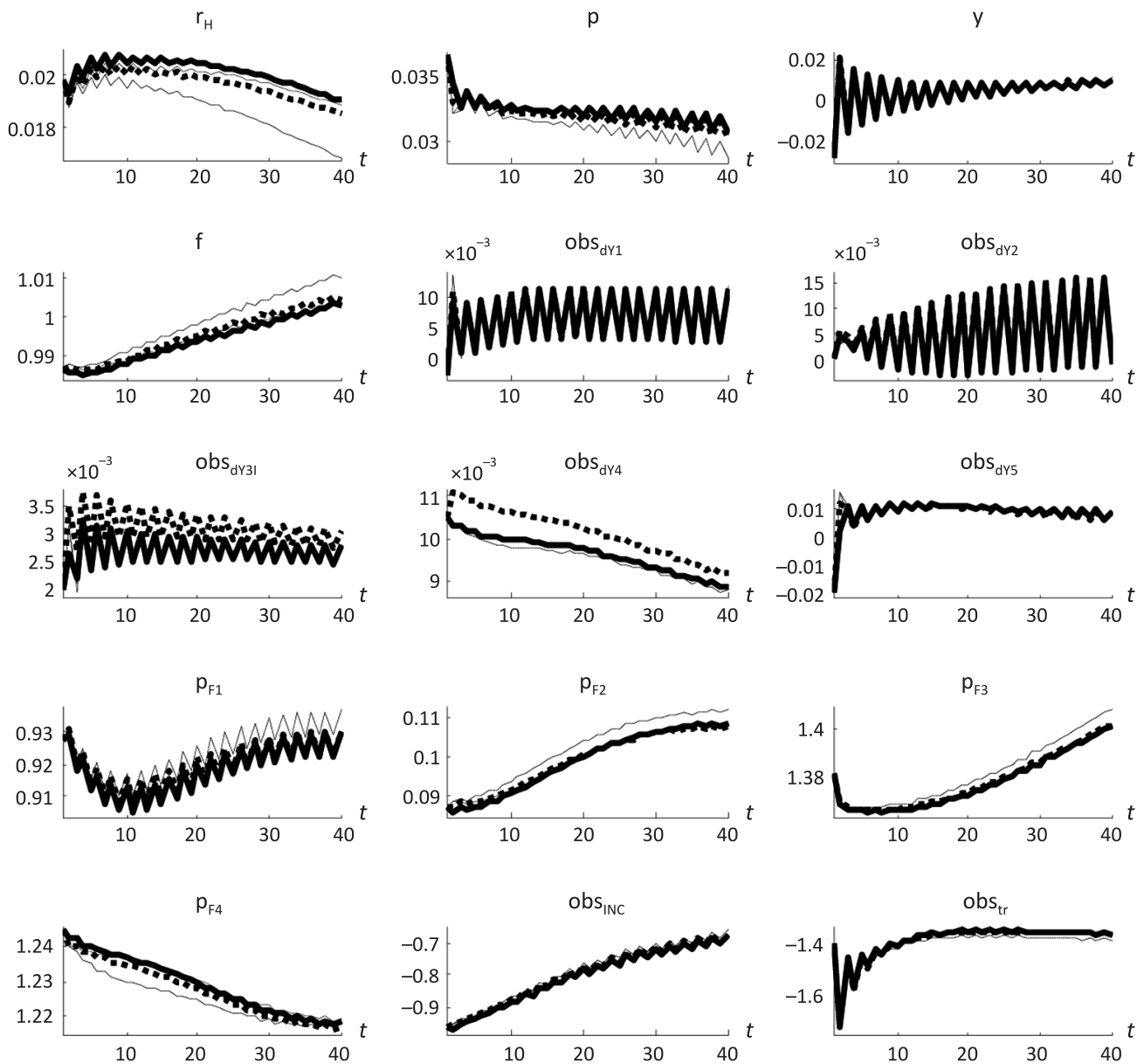

Puc. A2. Последствия ограничения доступа фирм к валютному финансированию Вертикальная ось: значение переменной. Горизонтальная ось: время в кварталах Обозначения: прогноз ——; изменение параметров ......; одномоментный шок . . . . . ; перманентный шок - - - - - - . 
$r_{H}$

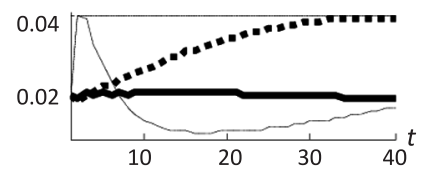

f
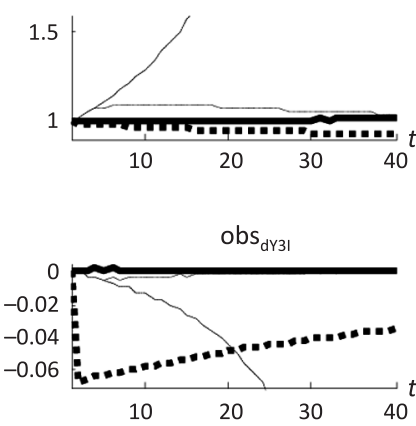

$\mathrm{p}_{\mathrm{F} 1}$

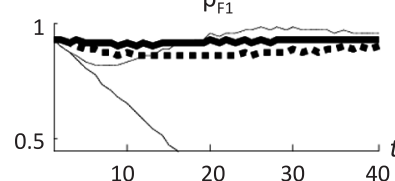

$p_{F 4}$

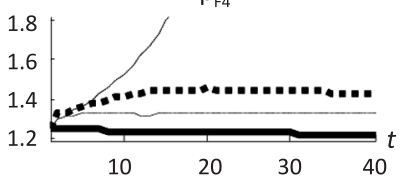

p

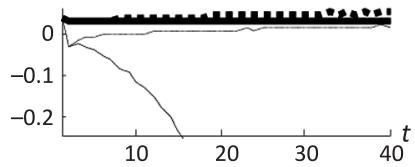

$\mathrm{obs}_{\mathrm{dY} 1}$
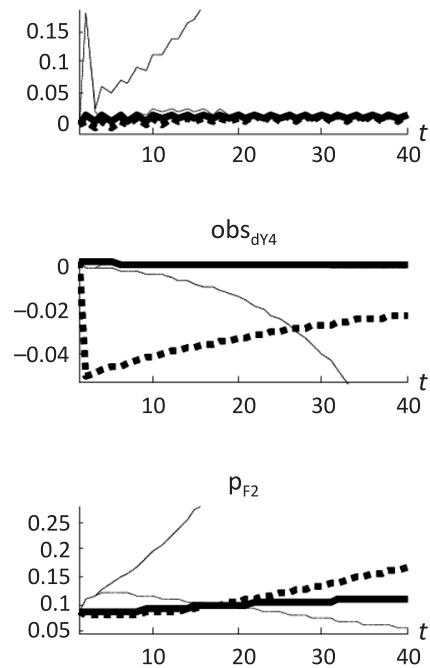

obs $_{\text {INC }}$

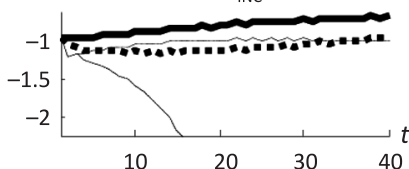

y

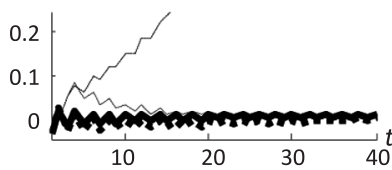

$\mathrm{obs}_{\mathrm{dY} 2}$
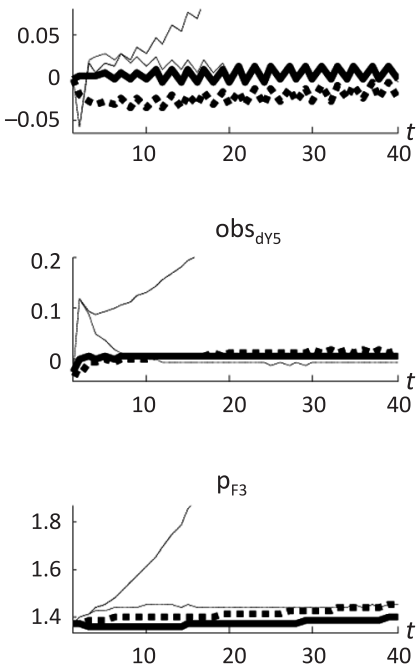

$\mathrm{obs}_{\mathrm{tr}}$

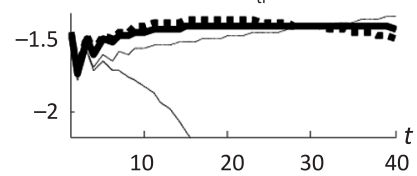

Puc. A3. Последствия роста ставок на $2 \%$

Вертикальная ось: значение переменной. Горизонтальная ось: время в кварталах

Обозначения: прогноз ——; изменение параметров .......; одномоментный шок .......; перманентный шок - - - - - -. 
$r_{H}$

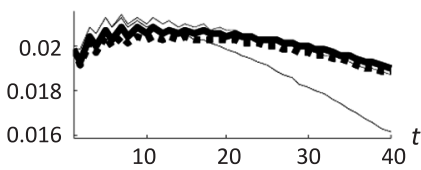

$f$
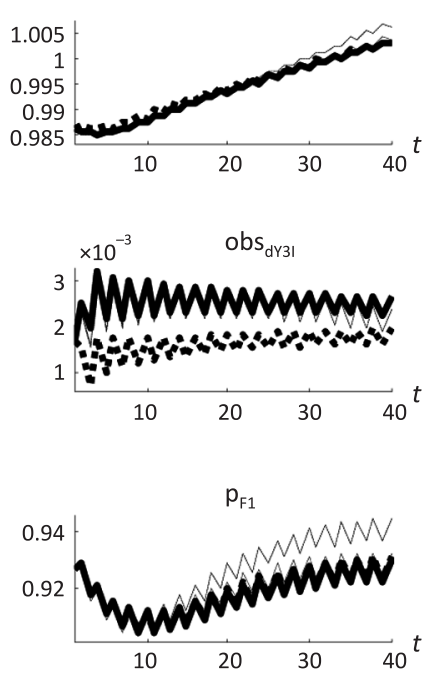

$p_{F 4}$

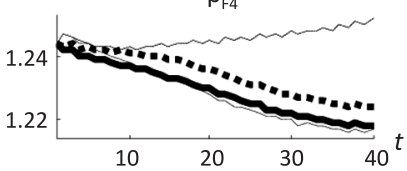

p
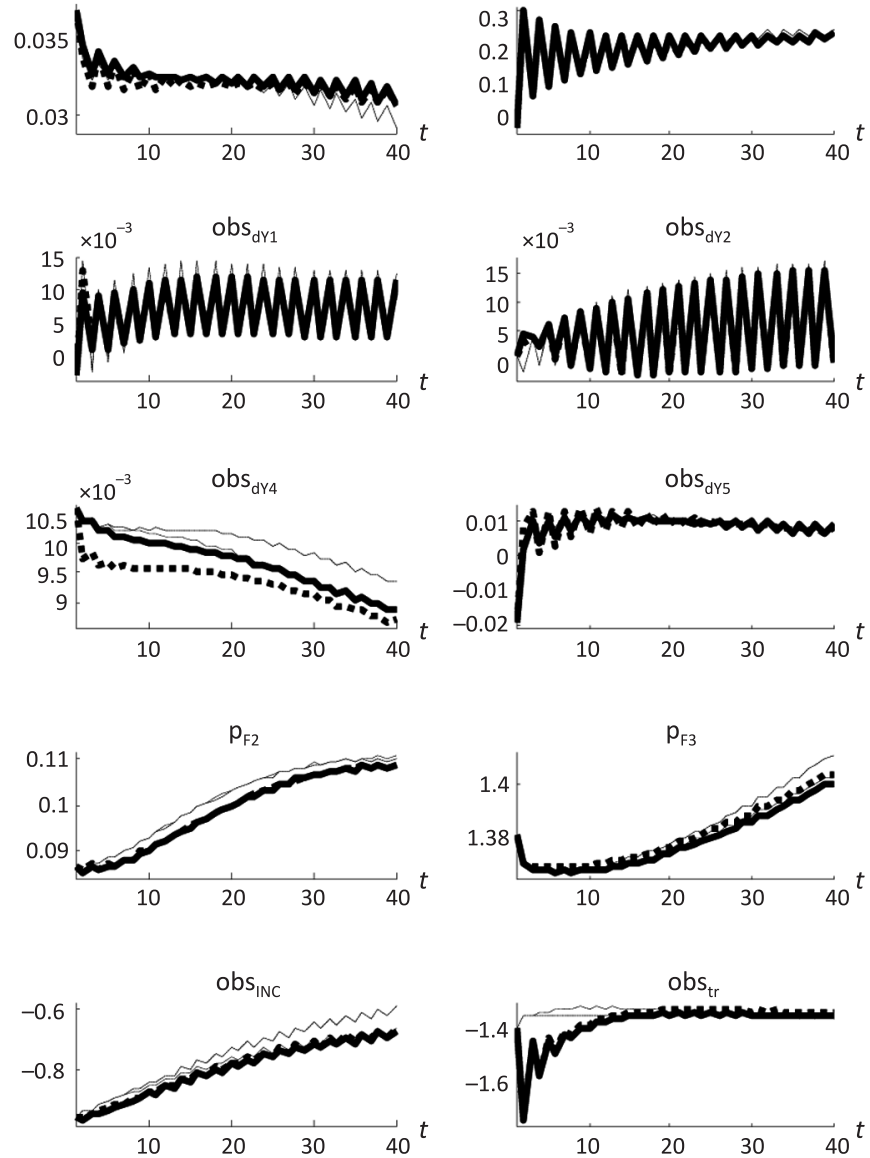

Puc. A4. Последствия повышения государственных расходов и трансфертов Вертикальная ось: значение переменной. Горизонтальная ось: время в кварталах

Обозначения: прогноз ——; изменение параметров . . . . . . ; одномоментный шок . . . . . .; перманентный шок _ - . . . . - .

Статья поступила в редакцию 6 декабря 2015 г. Статья рекомендована в печать 7 сентября 2016 г. 